\title{
Predual Spaces of Banach Completions of Orlicz-Hardy Spaces Associated with Operators
}

\author{
Renjin Jiang and Dachun Yang*
}

\begin{abstract}
Let $L$ be a linear operator in $L^{2}\left(\mathbb{R}^{n}\right)$ and generate an analytic semigroup $\left\{e^{-t L}\right\}_{t \geq 0}$ with kernels satisfying an upper bound of Poisson type, whose decay is measured by $\theta(L) \in(0, \infty]$. Let $\omega$ on $(0, \infty)$ be of upper type 1 and of critical lower type $\widetilde{p}_{0}(\omega) \in(n /(n+\theta(L)), 1]$ and $\rho(t)=t^{-1} / \omega^{-1}\left(t^{-1}\right)$ for $t \in(0, \infty)$. In this paper, the authors first introduce the VMO-type space $\operatorname{VMO}_{\rho, L}\left(\mathbb{R}^{n}\right)$ and the tent space $T_{\omega, \mathrm{v}}^{\infty}\left(\mathbb{R}_{+}^{n+1}\right)$ and characterize the space $\operatorname{VMO}_{\rho, L}\left(\mathbb{R}^{n}\right)$ via the space $T_{\omega, \mathrm{v}}^{\infty}\left(\mathbb{R}_{+}^{n+1}\right)$. Let $\widetilde{T}_{\omega}\left(\mathbb{R}_{+}^{n+1}\right)$ be the Banach completion of the tent space $T_{\omega}\left(\mathbb{R}_{+}^{n+1}\right)$. The authors then prove that $\widetilde{T}_{\omega}\left(\mathbb{R}_{+}^{n+1}\right)$ is the dual space of $T_{\omega, \mathrm{v}}^{\infty}\left(\mathbb{R}_{+}^{n+1}\right)$. As an application of this, the authors finally show that the dual space of $\operatorname{VMO}_{\rho, L^{*}}\left(\mathbb{R}^{n}\right)$ is the space $B_{\omega, L}\left(\mathbb{R}^{n}\right)$, where $L^{*}$ denotes the adjoint operator of $L$ in $L^{2}\left(\mathbb{R}^{n}\right)$ and $B_{\omega, L}\left(\mathbb{R}^{n}\right)$ the Banach completion of the Orlicz-Hardy space $H_{\omega, L}\left(\mathbb{R}^{n}\right)$. These results generalize the known recent results by particularly taking $\omega(t)=t$ for $t \in(0, \infty)$.
\end{abstract}

\section{Introduction}

The space $\operatorname{VMO}\left(\mathbb{R}^{n}\right)$ (the space of functions with vanishing mean oscillation) was first studied by Sarason [21]. Coifman and Weiss [4] introduced the space $\operatorname{CMO}\left(\mathbb{R}^{n}\right)$ which is defined to be the closure in the BMO norm of the space of continuous functions with compact support, and moreover, proved that the space $\operatorname{CMO}\left(\mathbb{R}^{n}\right)$ is the predual of the Hardy space $H^{1}\left(\mathbb{R}^{n}\right)$. When $p<1$, Janson [16] introduced the space $\lambda_{n(1 / p-1)}\left(\mathbb{R}^{n}\right)$ which is defined to be the closure of the space of Schwartz functions in the norm of the Lipschitz space $\Lambda_{n(1 / p-1)}\left(\mathbb{R}^{n}\right)$, and proved that $\left(\lambda_{n(1 / p-1)}\left(\mathbb{R}^{n}\right)\right)^{*}=B^{p}\left(\mathbb{R}^{n}\right)$, where $B^{p}\left(\mathbb{R}^{n}\right)$ is the Banach completion of the Hardy space $H^{p}\left(\mathbb{R}^{n}\right)$; see also [20, 23] for more properties about the space $\lambda_{n(1 / p-1)}\left(\mathbb{R}^{n}\right)$.

In recent years, the study of function spaces associated with operators has inspired great interests; see, for example, $[1,6,7,10,11,12,15,24,25]$ and their references. Let $L$ be a linear operator in $L^{2}\left(\mathbb{R}^{n}\right)$ and generate an analytic semigroup $\left\{e^{-t L}\right\}_{t \geq 0}$ with kernels satisfying an upper bound of Poisson type, whose decay is measured by $\theta(L) \in(0, \infty]$. Auscher, Duong and McIntosh [1] introduced the Hardy space $H_{L}^{1}\left(\mathbb{R}^{n}\right)$ by using the Lusinarea function and established its molecular characterization. Duong and Yan $[11,13]$,

2000 Mathematics Subject Classification. Primary 42B35; Secondary 42B30.

Key words and phrases. operator, Orlicz function, Orlicz-Hardy space, VMO, predual space, Banach completion, tent space, molecule.

Dachun Yang is supported by the National Natural Science Foundation (Grant No. 10871025) of China.

* Corresponding author. 
and Duong, Xiao and Yan [10] introduced and studied some BMO spaces and MorreyCampanato spaces associated with operators. Duong and Yan [12] further proved that the dual space of the Hardy space $H_{L}^{1}\left(\mathbb{R}^{n}\right)$ is the space $\mathrm{BMO}_{L^{*}}\left(\mathbb{R}^{n}\right)$ introduced in [11], where $L^{*}$ denotes the adjoint operator of $L$ in $L^{2}\left(\mathbb{R}^{n}\right)$. Yan [25] generalized all these results to the Hardy spaces $H_{L}^{p}\left(\mathbb{R}^{n}\right)$ with $p \in(n /(n+\theta(L)), 1]$ and their dual spaces. Moreover, recently, Deng, Duong et al in [6] introduced the space $\mathrm{VMO}_{L}\left(\mathbb{R}^{n}\right)$ and proved that $\left(\mathrm{VMO}_{L^{*}}\left(\mathbb{R}^{n}\right)\right)^{*}=H_{L}^{1}\left(\mathbb{R}^{n}\right)$.

On the other hand, the Orlicz-Hardy space was studied by Janson [16] and Viviani [22]. Let $\omega$ on $(0, \infty)$ be of upper type 1 and of critical lower type $\widetilde{p}_{0}(\omega) \in(n /(n+\theta(L)), 1]$ and $\rho(t) \equiv t^{-1} / \omega^{-1}\left(t^{-1}\right)$ for $t \in(0, \infty)$. The Orlicz-Hardy space $H_{\omega, L}\left(\mathbb{R}^{n}\right)$ and its dual space $\mathrm{BMO}_{\rho, L^{*}}\left(\mathbb{R}^{n}\right)$ associated with the aforementioned operator $L$ and its dual operator $L^{*}$ in $L^{2}\left(\mathbb{R}^{n}\right)$ were introduced in [17]. If $\omega(t)=t^{p}$ for all $t \in(0, \infty)$, then $H_{\omega, L}\left(\mathbb{R}^{n}\right)=H_{L}^{p}\left(\mathbb{R}^{n}\right)$ and $\mathrm{BMO}_{\rho, L^{*}}\left(\mathbb{R}^{n}\right)$ becomes $\mathrm{BMO}_{L^{*}}\left(\mathbb{R}^{n}\right)$ when $p=1$ or the Morrey-Campanato space (see [13]) when $p<1$. The main purpose of this paper is to study the predual space of the Banach completion of the Orlicz-Hardy space $H_{\omega, L}\left(\mathbb{R}^{n}\right)$.

In fact, in this paper, we first introduce the VMO-type space $\mathrm{VMO}_{\rho, L}\left(\mathbb{R}^{n}\right)$ and the tent space $T_{\omega, \mathrm{v}}^{\infty}\left(\mathbb{R}_{+}^{n+1}\right)$ and characterize the space $\mathrm{VMO}_{\rho, L}\left(\mathbb{R}^{n}\right)$ via the space $T_{\omega, \mathrm{v}}^{\infty}\left(\mathbb{R}_{+}^{n+1}\right)$. Let $\widetilde{T}_{\omega}\left(\mathbb{R}_{+}^{n+1}\right)$ be the Banach completion of the tent space $T_{\omega}\left(\mathbb{R}_{+}^{n+1}\right)$. We then prove that $\widetilde{T}_{\omega}\left(\mathbb{R}_{+}^{n+1}\right)$ is the dual space of $T_{\omega, \mathrm{v}}^{\infty}\left(\mathbb{R}_{+}^{n+1}\right)$. As an application of this, we finally show that the dual space of $\operatorname{VMO}_{\rho, L^{*}}\left(\mathbb{R}^{n}\right)$ is the space $B_{\omega, L}\left(\mathbb{R}^{n}\right)$, where $L^{*}$ denotes the adjoint operator of $L$ in $L^{2}\left(\mathbb{R}^{n}\right)$ and $B_{\omega, L}\left(\mathbb{R}^{n}\right)$ the Banach completion of the Orlicz-Hardy space $H_{\omega, L}\left(\mathbb{R}^{n}\right)$. In particular, if $p \in(0,1]$ and $\omega(t)=t^{p}$ for all $t \in(0, \infty)$, we obtain the predual space of the Banach completion of the Hardy space $H_{L}^{p}\left(\mathbb{R}^{n}\right)$ in [25], and if $p=1$, we re-obtain that $\left(\mathrm{VMO}_{L^{*}}\left(\mathbb{R}^{n}\right)\right)^{*}=H_{L}^{1}\left(\mathbb{R}^{n}\right)$, which is the main result in [6]. Moreover, we prove that if $L=\Delta, p \in(0,1]$ and $\omega(t)=t^{p}$ for all $t \in(0, \infty)$, the space $\operatorname{VMO}_{\rho, L}\left(\mathbb{R}^{n}\right)$ coincides with the space $\lambda_{n(1 / p-1)}\left(\mathbb{R}^{n}\right)$ in [16] (see also $[20,23]$ ), where $\Delta=-\sum_{i=1}^{n} \frac{\partial^{2}}{\partial x_{i}^{2}}$ is the Laplace operator on $\mathbb{R}^{n}$.

Precisely, this paper is organized as follows. In Section 2, we recall some known definitions and notation concerning aforementioned operators, Orlicz functions, the OrliczHardy spaces and BMO spaces associated with these operators and describe some basic assumptions on the operator $L$ and the Orlicz function $\omega$ considered in this paper. We remark that there exist many operators satisfying these assumptions (see $[6,10,12,25,17]$ for examples of such operators). Also, if $p \in(0,1]$, then $\omega(t)=t^{p}$ for all $t \in(0, \infty)$ is a typical example of Orlicz functions satisfying our assumptions; see [17] for some other examples.

In Section 3, we introduce the spaces $\operatorname{VMO}_{\rho, L}\left(\mathbb{R}^{n}\right)$ and $T_{\omega, \mathrm{v}}^{\infty}\left(\mathbb{R}_{+}^{n+1}\right)$ and give some basic properties of these spaces. In particular, we characterize the space $\operatorname{VMO}_{\rho, L}\left(\mathbb{R}^{n}\right)$ via the space $T_{\omega, \mathrm{V}}^{\infty}\left(\mathbb{R}_{+}^{n+1}\right)$; see Theorem 3.2 below. As an application of Theorem 3.2 together with a characterization of the space $\lambda_{n(1 / p-1)}\left(\mathbb{R}^{n}\right)$ in $[23]$, we obtain that when $L=\Delta$, $p \in(0,1]$ and $\omega(t)=t^{p}$ for all $t \in(0, \infty)$, the space $\operatorname{VMO}_{\rho, L}\left(\mathbb{R}^{n}\right)$ coincides with the space $\lambda_{n(1 / p-1)}\left(\mathbb{R}^{n}\right)$; see Corollary 3.1 below.

In Section 4, we introduce the space $\widetilde{T}_{\omega}\left(\mathbb{R}_{+}^{n+1}\right)$, which is defined to be the Banach completion of the tent space $T_{\omega}\left(\mathbb{R}_{+}^{n+1}\right)$ (see Definition 4.1 below), and prove that $\widetilde{T}_{\omega}\left(\mathbb{R}_{+}^{n+1}\right)$ 
is the dual space of $T_{\omega, \mathrm{v}}^{\infty}\left(\mathbb{R}_{+}^{n+1}\right)$; see Theorem 4.2 below. If $p \in(0,1]$ and $\omega(t)=t^{p}$ for all $t \in(0, \infty)$, then the tent space $T_{\omega}\left(\mathbb{R}_{+}^{n+1}\right)=T_{2}^{p}\left(\mathbb{R}_{+}^{n+1}\right)$ and its predual space was proved to be the corresponding space $T_{\omega, \mathrm{v}}^{\infty}\left(\mathbb{R}_{+}^{n+1}\right)$ by Wang in [23], which when $p=1$ plays a key role in $[6]$. To prove that $\widetilde{T}_{\omega}\left(\mathbb{R}_{+}^{n+1}\right)$ is the dual space of $T_{\omega, \mathrm{v}}^{\infty}\left(\mathbb{R}_{+}^{n+1}\right)$, different from the approach used in [4] and [23] which strongly depends on an unfamiliar result (Exercise 41 on [8, p. 439]) from the functional analysis, we only use the basic fact that the dual space of $L^{2}$ is itself. Indeed, using this fact, for any $\ell \in\left(T_{\omega, \mathrm{v}}^{\infty}\left(\mathbb{R}_{+}^{n+1}\right)\right)^{*}$, we construct $g \in \widetilde{T}_{\omega}\left(\mathbb{R}_{+}^{n+1}\right)$ such that for all $f \in T_{\omega, \mathrm{v}}^{\infty}\left(\mathbb{R}_{+}^{n+1}\right)$,

$$
\ell(f)=\int_{\mathbb{R}_{+}^{n+1}} f(x, t) g(x, t) \frac{d x d t}{t}
$$

see Theorem 4.2 below. As an application of Theorem 4.2, we further prove that the dual space of the space $\operatorname{VMO}_{\rho, L^{*}}\left(\mathbb{R}^{n}\right)$ is the space $B_{\omega, L}\left(\mathbb{R}^{n}\right)$, where the space $B_{\omega, L}\left(\mathbb{R}^{n}\right)$ is the Banach completion of $H_{\omega, L}\left(\mathbb{R}^{n}\right)$; see Definition 4.3 and Theorem 4.4 below. Since all dual spaces are complete, it is necessary here to replace the Orlicz-Hardy space by its Banach completion, which is different from [6]. In [6], the Banach completion of the Hardy space $H_{L}^{1}\left(\mathbb{R}^{n}\right)$ is just itself.

Finally, we make some conventions. Throughout the paper, we denote by $C$ a positive constant which is independent of the main parameters, but it may vary from line to line. The symbol $X \lesssim Y$ means that there exists a positive constant $C$ such that $X \leq C Y$; the symbol $\lfloor\alpha\rfloor$ for $\alpha \in \mathbb{R}$ denotes the maximal integer no more than $\alpha ; B \equiv B\left(z_{B}, r_{B}\right)$ denotes an open ball with center $z_{B}$ and radius $r_{B}$ and $C B\left(z_{B}, r_{B}\right) \equiv B\left(z_{B}, C r_{B}\right)$. Set $\mathbb{N} \equiv\{1,2, \cdots\}$ and $\mathbb{Z}_{+} \equiv \mathbb{N} \cup\{0\}$. For any subset $E$ of $\mathbb{R}^{n}$, we denote by $E^{\complement}$ the set $\mathbb{R}^{n} \backslash E$.

\section{Preliminaries}

In this section, we first describe some basic assumptions on the operators $L$ and Orlicz functions studied in this paper (see, for example, $[9,18,16,22,10,11,12,6,17]$ ), and we then recall some notions about the Orlicz-Hardy space $H_{\omega, L}\left(\mathbb{R}^{n}\right)$ and the BMO-type space $\mathrm{BMO}_{\rho, L}\left(\mathbb{R}^{n}\right)$ in $[17]$.

\subsection{Two assumptions on the operator $L$}

Let $\nu \in(0, \pi), S_{\nu} \equiv\{z \in \mathbb{C}:|\arg (z)| \leq \nu\} \cup\{0\}$ and $S_{\nu}^{0}$ the interior of $S_{\nu}$, where $\arg (z) \in(-\pi, \pi]$ is the argument of $z$. Assume that $L$ is a linear operator such that $\sigma(L) \subset S_{\nu}$, where $\sigma(L)$ denotes the spectra of $L$ and $\nu \in(0, \pi / 2)$, and that for all $\gamma>\nu$, there exists a positive constant $C_{\gamma}$ such that

$$
\left\|(L-\lambda I)^{-1}\right\|_{L^{2}\left(\mathbb{R}^{n}\right) \rightarrow L^{2}\left(\mathbb{R}^{n}\right)} \leq C_{\gamma}|\lambda|^{-1}, \quad \forall \lambda \notin S_{\gamma},
$$

where and in what follows, for any two normed linear spaces $\mathscr{X}$ and $\mathscr{Y}$, and any bounded linear operator $T$ from $\mathscr{X}$ to $\mathscr{Y}$, we use $\|T\|_{\mathscr{X} \rightarrow \mathscr{Y}}$ to denote the operator norm of $T$ from $\mathscr{X}$ to $\mathscr{Y}$ and $\mathfrak{L}(\mathscr{X}, \mathscr{Y})$ the set of all bounded linear operators from $\mathscr{X}$ to $\mathscr{Y}$. Hence $L$ 
generates a holomorphic semigroup $e^{-z L}$, where $0 \leq|\arg (z)|<\frac{\pi}{2}-\nu$ (see [18]). We make the following two assumptions on $L$ (see $[12,10,6,25,17])$.

Assumption (a) Assume that for all $t>0$, the distribution kernels $p_{t}$ of $e^{-t L}$ belong to $L^{\infty}\left(\mathbb{R}^{n} \times \mathbb{R}^{n}\right)$ and satisfy the estimate $\left|p_{t}(x, y)\right| \leq\left|h_{t}(x, y)\right|$ for all $x, y \in \mathbb{R}^{n}$, where $h_{t}$ is given by

$$
h_{t}(x, y)=t^{-\frac{n}{m}} g\left(\frac{|x-y|}{t^{\frac{1}{m}}}\right)
$$

in which $m$ is a positive constant and $g$ is a positive, bounded, decreasing function satisfying

$$
\lim _{r \rightarrow \infty} r^{n+\epsilon} g(r)=0
$$

for some $\epsilon>0$.

Let $H\left(S_{\nu}^{0}\right)$ be the space of all holomorphic functions on $S_{\nu}^{0}$ and

$$
H_{\infty}\left(S_{\gamma}^{0}\right) \equiv\left\{b \in H\left(S_{\gamma}^{0}\right):\|b\|_{\infty} \equiv \sup _{z \in S_{\gamma}^{0}}|b(z)|<\infty\right\} .
$$

Recall that the operator $L$ is said to have a bounded $H_{\infty}$-calculus in $L^{2}\left(\mathbb{R}^{n}\right)$ (see [18]) provided that for all $\gamma \in(\nu, \pi)$, there exists a positive constant $\widetilde{C}_{\gamma}$ such that for all $b \in$ $H_{\infty}\left(S_{\gamma}^{0}\right), b(L) \in \mathfrak{L}\left(L^{2}\left(\mathbb{R}^{n}\right), L^{2}\left(\mathbb{R}^{n}\right)\right)$ and $\|b(L)\|_{L^{2}\left(\mathbb{R}^{n}\right) \rightarrow L^{2}\left(\mathbb{R}^{n}\right)} \leq \widetilde{C}_{\gamma}\|b\|_{\infty}$, where $\psi(z)=$ $z(1+z)^{-2}$ for all $z \in S_{\gamma}^{0}$ and $b(L) \equiv[\psi(L)]^{-1}(b \psi)(L)$. It was proved in [18] that $b(L)$ is a well-defined linear operator in $L^{2}\left(\mathbb{R}^{n}\right)$.

Assumption (b) Assume that the operator $L$ is one-to-one, has dense range in $L^{2}\left(\mathbb{R}^{n}\right)$ and a bounded $H_{\infty}$-calculus in $L^{2}\left(\mathbb{R}^{n}\right)$.

From the assumptions (a) and (b), it is easy to deduce the following useful estimates.

First, if $\left\{e^{-t L}\right\}_{t \geq 0}$ is a bounded analytic semigroup in $L^{2}\left(\mathbb{R}^{n}\right)$ whose kernels $\left\{p_{t}\right\}_{t \geq 0}$ satisfy the estimates (2.1) and (2.2), then for any $k \in \mathbb{N}$, there exists a positive constant $C$ such that the time derivatives of $p_{t}$ satisfy

$$
\left|t^{k} \frac{\partial^{k} p_{t}(x, y)}{\partial t^{k}}\right| \leq \frac{C}{t^{\frac{n}{m}}} g\left(\frac{|x-y|}{t^{\frac{1}{m}}}\right)
$$

for all $t>0$ and almost everywhere $x, y \in \mathbb{R}^{n}$. It should be pointed out that for any $k \in \mathbb{N}$, the function $g$ may depend on $k$ but it always satisfies (2.2); see Theorem 6.17 of [19] and [5], and also [12, 10, 6, 25, 17].

Secondly, let

$$
\Psi\left(S_{\nu}^{0}\right) \equiv\left\{\psi \in H\left(S_{\nu}^{0}\right): \exists s, C>0 \text { such that } \forall z \in S_{\nu}^{0},|\psi(z)| \leq C|z|^{s}\left(1+|z|^{2 s}\right)^{-1}\right\} .
$$


It is well known that $L$ has a bounded $H_{\infty}$-calculus in $L^{2}\left(\mathbb{R}^{n}\right)$ if and only if for all $\gamma \in(\nu, \pi]$ and any non-zero function $\psi \in \Psi\left(S_{\gamma}^{0}\right), L$ satisfies the square function estimate and its reverse, namely, there exists a positive constant $C$ such that for all $f \in L^{2}\left(\mathbb{R}^{n}\right)$,

$$
C^{-1}\|f\|_{L^{2}\left(\mathbb{R}^{n}\right)} \leq\left(\int_{0}^{\infty}\left\|\psi_{t}(L) f\right\|_{L^{2}\left(\mathbb{R}^{n}\right)}^{2} \frac{d t}{t}\right)^{1 / 2} \leq C\|f\|_{L^{2}\left(\mathbb{R}^{n}\right)},
$$

where $\psi_{t}(\xi)=\psi(t \xi)$ for all $t>0$ and $\xi \in \mathbb{R}^{n}$. Notice that different choices of $\gamma>\nu$ and $\psi \in \Psi\left(S_{\gamma}^{0}\right)$ lead to equivalent quadratic norms of $f$; see [18] for the details.

As noticed in [18], positive self-adjoint operators satisfy the quadratic estimate (2.4). So do normal operators with spectra in a sector, and maximal accretive operators. For definitions of these classes of operators, we refer the reader to [26].

\subsection{An acting class of the semigroup $\left\{e^{-t L}\right\}_{t \geq 0}$}

Duong and Yan [11] introduced the class of functions that the operators $e^{-t L}$ act upon. Precisely, for any $\beta>0$, let $\mathcal{M}_{\beta}\left(\mathbb{R}^{n}\right)$ be the collection of all functions $f \in L_{\text {loc }}^{2}\left(\mathbb{R}^{n}\right)$ such that

$$
\|f\|_{\mathcal{M}_{\beta}\left(\mathbb{R}^{n}\right)} \equiv\left(\int_{\mathbb{R}^{n}} \frac{|f(x)|^{2}}{1+|x|^{n+\beta}} d x\right)^{1 / 2}<\infty .
$$

Then $\mathcal{M}_{\beta}\left(\mathbb{R}^{n}\right)$ is a Banach space under the norm $\|\cdot\|_{\mathcal{M}_{\beta}\left(\mathbb{R}^{n}\right)}$. For any given operator $L$, set

$$
\theta(L) \equiv \sup \{\epsilon>0:(2.2) \text { holds }\}
$$

and define

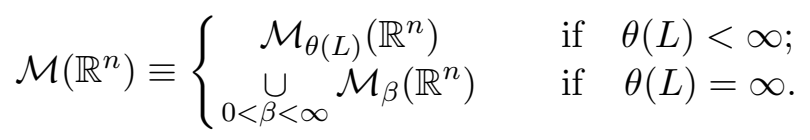

Let $s \in \mathbb{Z}_{+}$. For any $(x, t) \in \mathbb{R}_{+}^{n+1} \equiv \mathbb{R}^{n} \times(0, \infty)$ and $f \in \mathcal{M}\left(\mathbb{R}^{n}\right)$, set

$$
P_{s, t} f(x) \equiv f(x)-\left(I-e^{-t L}\right)^{s+1} f(x) \quad \text { and } \quad Q_{s, t} f(x) \equiv t^{s+1} L^{s+1} e^{-t L} f(x) .
$$

If $s=0$, write

$$
P_{t} f(x) \equiv P_{0, t} f(x)=e^{-t L} f(x) \quad \text { and } \quad Q_{t} f(x) \equiv Q_{0, t} f(x)=t L e^{-t L} f(x) .
$$

For any $f \in \mathcal{M}\left(\mathbb{R}^{n}\right)$, by (2.3), it is easy to show that $P_{s, t} f$ and $Q_{s, t} f$ are well defined. Moreover, by (2.3) again, we know that the kernels $p_{s, t}$ and $q_{s, t}$ of $P_{s, t}$ of $Q_{s, t}$ satisfy that for all $t>0$ and $x, y \in \mathbb{R}^{n}$,

$$
\left|p_{s, t^{m}}(x, y)\right|+\left|q_{s, t^{m}}(x, y)\right| \leq C_{s} t^{-n} g\left(\frac{|x-y|}{t}\right),
$$

where the function $g$ satisfies the condition $(2.2)$ and $C_{s}$ is a positive constant independent of $t, x$ and $y$.

It should be pointed out that these operators in (2.6) were introduced by Blunck and Kunstmann [2]. 


\subsection{Orlicz functions}

Let $\omega$ be a positive function defined on $\mathbb{R}_{+} \equiv(0, \infty)$. The function $\omega$ is said to be of upper type $p$ (resp. lower type $p$ ) for some $p \in[0, \infty)$, if there exists a positive constant $C$ such that for all $t \geq 1$ (resp. $0<t \leq 1$ ),

$$
\omega(s t) \leq C t^{p} \omega(s)
$$

Obviously, if $\omega$ is of lower type $p$ for some $p>0$, then $\lim _{t \rightarrow 0^{+}} \omega(t)=0$. So for the sake of convenience, if it is necessary, we may assume that $\omega(0)=0$. If $\omega$ is of both upper type $p_{1}$ and lower type $p_{0}$, then $\omega$ is said to be of type $\left(p_{0}, p_{1}\right)$. Let

$$
\widetilde{p}_{1}(\omega) \equiv \inf \{p>0:(2.9) \text { holds for all } t \in(1, \infty)\}
$$

and

$$
\widetilde{p}_{0}(\omega) \equiv \sup \{p>0:(2.9) \text { holds for all } t \in(0,1)\} .
$$

It is easy to see that $\widetilde{p}_{0}(\omega) \leq \widetilde{p}_{1}(\omega)$ for all $\omega$. In what follows, $\widetilde{p}_{0}(\omega)$ and $\widetilde{p}_{1}(\omega)$ are called the critical lower type index and the critical upper type index of $\omega$, respectively. Throughout the whole paper, we always assume that $\omega$ satisfies the following assumption.

Assumption (c) Suppose that the positive Orlicz function $\omega$ on $\mathbb{R}_{+}$is continuous, strictly increasing, subadditive, of upper type 1 and $\widetilde{p}_{0}(\omega) \in\left(\frac{n}{n+\theta(L)}, 1\right]$, where $\theta(L)$ is as in (2.5).

Notice that for any $\omega$ of type $\left(p_{0}, p_{1}\right)$, if we set $\widetilde{\omega}(t) \equiv \int_{0}^{t} \frac{\omega(s)}{s} d s$ for $t \in[0, \infty)$, then by [22, Proposition 3.1], $\widetilde{\omega}$ is equivalent to $\omega$, namely, there exists a positive constant $C$ such that $C^{-1} \omega(t) \leq \widetilde{\omega}(t) \leq C \omega(t)$ for all $t \in[0, \infty)$, and moreover, $\widetilde{\omega}$ is strictly increasing, subadditive and continuous function of type $\left(p_{0}, p_{1}\right)$. Since all our results in this paper are invariant on equivalent functions, we always assume that $\omega$ satisfies the assumption (c); otherwise, we may replace $\omega$ by $\widetilde{\omega}$.

We also make the following convention.

Convention From the assumption (c), it follows that $\frac{n}{n+\theta(L)}<\widetilde{p}_{0}(\omega) \leq \widetilde{p}_{1}(\omega) \leq 1$. In what follows, if $(2.9)$ holds for $\widetilde{p}_{1}(\omega)$ with $t \in(1, \infty)$, then we choose $p_{1}(\omega) \equiv \widetilde{p}_{1}(\omega)$; otherwise $\widetilde{p}_{1}(\omega)<1$ and we choose $p_{1}(\omega) \in\left(\widetilde{p}_{1}(\omega), 1\right)$. Similarly, if $(2.9)$ holds for $\widetilde{p}_{0}(\omega)$ with $t \in(0,1)$, then we choose $p_{0}(\omega) \equiv \widetilde{p}_{0}(\omega)$; otherwise we choose $p_{0}(\omega) \in\left(\frac{n}{n+\theta(L)}, \widetilde{p}_{0}(\omega)\right)$ such that $\left\lfloor\frac{n}{m}\left(\frac{1}{p_{0}(\omega)}-1\right)\right\rfloor=\left\lfloor\frac{n}{m}\left(\frac{1}{\tilde{p}_{0}(\omega)}-1\right)\right\rfloor$, where $m$ is as in $(2.1)$.

Let $\omega$ satisfy the assumption (c). A measurable function $f$ on $\mathbb{R}^{n}$ is said to be in the Lebesgue type space $L(\omega)$ if $\int_{\mathbb{R}^{n}} \omega(|f(x)|) d x<\infty$. Moreover, for any $f \in L(\omega)$, define

$$
\|f\|_{L(\omega)} \equiv \inf \left\{\lambda>0: \int_{\mathbb{R}^{n}} \omega\left(\frac{|f(x)|}{\lambda}\right) d x \leq 1\right\}
$$


Let $\omega$ satisfy the assumption (c). Define the function $\rho(t)$ on $\mathbb{R}_{+}$by setting, for all $t \in(0, \infty)$

$$
\rho(t) \equiv \frac{t^{-1}}{\omega^{-1}\left(t^{-1}\right)}
$$

where $\omega^{-1}$ is the inverse function of $\omega$. Then by Proposition 2.1 in [17], $\rho$ is of type $\left(1 / p_{1}(\omega)-1,1 / p_{0}(\omega)-1\right)$, which is denoted by $\left(\beta_{0}(\rho), \beta_{1}(\rho)\right)$ in what follows for short.

\subsection{The Orlicz-Hardy space $H_{\omega, L}\left(\mathbb{R}^{n}\right)$ and its dual space}

For any function $f \in L^{1}\left(\mathbb{R}^{n}\right)$, the Lusin area function $\mathcal{S}_{L}(f)$ associated with the operator $L$ is defined by setting, for all $x \in \mathbb{R}^{n}$,

$$
\mathcal{S}_{L}(f)(x) \equiv\left(\int_{\Gamma(x)}\left|Q_{t^{m}} f(y)\right|^{2} \frac{d y d t}{t^{n+1}}\right)^{1 / 2}
$$

where $Q_{t^{m}}$ is as in (2.7). From the assumption (b) together with (2.4), it is easy to deduce that the Lusin area function $\mathcal{S}_{L}$ is bounded on $L^{2}\left(\mathbb{R}^{n}\right)$. Auscher, Duong and McIntosh [1] proved that for any $p \in(1, \infty)$, there exists a positive constant $C_{p}$ such that for all $f \in L^{p}\left(\mathbb{R}^{n}\right)$,

$$
C_{p}^{-1}\|f\|_{L^{p}\left(\mathbb{R}^{n}\right)} \leq\left\|\mathcal{S}_{L}(f)\right\|_{L^{p}\left(\mathbb{R}^{n}\right)} \leq C_{p}\|f\|_{L^{p}\left(\mathbb{R}^{n}\right)} ;
$$

see also Duong and McIntosh [9] and Yan [24]. By duality, the operator $S_{L^{*}}$ also satisfies the estimate (2.11), where $L^{*}$ is the adjoint operator of $L$ in $L^{2}\left(\mathbb{R}^{n}\right)$.

Recall that the Orlicz-Hardy space $H_{\omega, L}\left(\mathbb{R}^{n}\right)$ and the BMO-type space $\mathrm{BMO}_{\rho, L}\left(\mathbb{R}^{n}\right)$ were introduced in [17].

Definition 2.1 Let $L$ satisfy the assumptions (a) and (b) and $\omega$ satisfy the assumption (c). A function $f \in L^{2}\left(\mathbb{R}^{n}\right)$ is said to be in $\widetilde{H}_{\omega, L}\left(\mathbb{R}^{n}\right)$ if $\mathcal{S}_{L}(f) \in L(\omega)$, and moreover, define

$$
\|f\|_{H_{\omega, L}\left(\mathbb{R}^{n}\right)} \equiv\left\|\mathcal{S}_{L}(f)\right\|_{L(\omega)}=\inf \left\{\lambda>0: \int_{\mathbb{R}^{n}} \omega\left(\frac{\mathcal{S}_{L}(f)(x)}{\lambda}\right) d x \leq 1\right\} .
$$

The Orlicz-Hardy space $H_{\omega, L}\left(\mathbb{R}^{n}\right)$ associated with the operator $L$ is defined to be the completion of $\widetilde{H}_{\omega, L}\left(\mathbb{R}^{n}\right)$ in the norm $\|\cdot\|_{H_{\omega, L}\left(\mathbb{R}^{n}\right)}$.

Definition 2.2 Let L satisfy the assumptions (a) and (b), $\omega$ satisfy the assumption (c), $\rho$ be as in $(2.10), q \in[1, \infty)$ and $s \geq\left\lfloor\frac{n}{m}\left(\frac{1}{\tilde{p}_{0}(\omega)}-1\right)\right\rfloor$. A function $f \in \mathcal{M}\left(\mathbb{R}^{n}\right)$ is said to be in $\mathrm{BMO}_{\rho, L}^{q, s}\left(\mathbb{R}^{n}\right)$ if

$$
\|f\|_{\mathrm{BMO}_{\rho, L}^{q, s}\left(\mathbb{R}^{n}\right)} \equiv \sup _{B \subset \mathbb{R}^{n}} \frac{1}{\rho(|B|)}\left[\frac{1}{|B|} \int_{B}\left|f(x)-P_{s, t_{B}} f(x)\right|^{q} d x\right]^{1 / q}<\infty,
$$

where the supremum is taken over all balls $B$ of $\mathbb{R}^{n}$. 
Remark 2.1 (i) Let $p \in(0,1], q \in[1, \infty)$ and $s \geq\left\lfloor\frac{n}{m}\left(\frac{1}{p}-1\right)\right\rfloor$. If $\omega(t)=t$ for all $t \in(0, \infty)$, then $H_{\omega, L}\left(\mathbb{R}^{n}\right)=H_{L}^{1}\left(\mathbb{R}^{n}\right)$ and $\mathrm{BMO}_{\rho, L}^{q, s}\left(\mathbb{R}^{n}\right)=\mathrm{BMO}_{L}\left(\mathbb{R}^{n}\right)$, where $H_{L}^{1}\left(\mathbb{R}^{n}\right)$ and $\mathrm{BMO}_{L}\left(\mathbb{R}^{n}\right)$ were introduced by Duong and Yan [11, 12], respectively. If $p \in(n /(n+\theta(L)), 1)$ and $\omega(t)=t^{p}$ for all $t \in(0, \infty)$, then $H_{\omega, L}\left(\mathbb{R}^{n}\right)=H_{L}^{p}\left(\mathbb{R}^{n}\right)$ and $\mathrm{BMO}_{\rho, L}^{q, s}\left(\mathbb{R}^{n}\right)=\mathfrak{L}_{L}(1 / p-1, q, s)$, where $H_{L}^{p}\left(\mathbb{R}^{n}\right)$ and $\mathfrak{L}_{L}(1 / p-1, q, s)$ were introduced by Yan [25] and Duong and Yan [13], respectively.

(ii) For $q \in[1, \infty)$ and $s \geq s_{0} \equiv\left\lfloor\frac{n}{m}\left(\frac{1}{\widetilde{p}_{0}(\omega)}-1\right)\right\rfloor$, the spaces $\mathrm{BMO}_{\rho, L}^{q, s}\left(\mathbb{R}^{n}\right)$ coincide with $\mathrm{BMO}_{\rho, L}^{2, s_{0}}\left(\mathbb{R}^{n}\right)$; see Corollary 3.1 and Remark 4.4 of [17]. Hence, in what follows, we denote the space $\mathrm{BMO}_{\rho, L}^{q, s}\left(\mathbb{R}^{n}\right)$ simply by $\mathrm{BMO}_{\rho, L}\left(\mathbb{R}^{n}\right)$.

(iii) It was proved in [17] that the dual space of $H_{\omega, L}\left(\mathbb{R}^{n}\right)$ is the space $\mathrm{BMO}_{\rho, L^{*}}\left(\mathbb{R}^{n}\right)$, where $L^{*}$ denotes the adjoint operator of $L$ in $L^{2}\left(\mathbb{R}^{n}\right)$.

\section{$3 \quad \mathrm{VMO}_{\rho, L}\left(\mathbb{R}^{n}\right)$-type spaces}

Suppose that the assumptions (a), (b) and (c) hold. In this section, we study the spaces of functions with vanishing mean oscillation associated with operators and Orlicz functions. We begin with some notions and notation.

Definition 3.1 Let $L$ satisfy the assumptions (a) and (b), $\omega$ satisfy the assumption (c), $\rho$ be as in (2.10) and $s \geq\left\lfloor\frac{n}{m}\left(\frac{1}{\tilde{p}_{0}(\omega)}-1\right)\right\rfloor$. A function $f \in \mathrm{BMO}_{\rho, L}\left(\mathbb{R}^{n}\right)$ is said to be in $\operatorname{VMO}_{\rho, L}^{s}\left(\mathbb{R}^{n}\right)$, if it satisfies the limiting conditions $\gamma_{1}(f)=\gamma_{2}(f)=\gamma_{3}(f)=0$, where

$$
\begin{aligned}
& \gamma_{1}(f) \equiv \lim _{c \rightarrow 0} \sup _{\text {ball } B: r_{B} \leq c}\left(\frac{1}{|B|[\rho(|B|)]^{2}} \int_{B}\left|f(x)-P_{s,\left(r_{B}\right)^{m}} f(x)\right|^{2} d x\right)^{1 / 2}, \\
& \gamma_{2}(f) \equiv \lim _{c \rightarrow \infty} \sup _{\text {ball } B: r_{B} \geq c}\left(\frac{1}{|B|[\rho(|B|)]^{2}} \int_{B}\left|f(x)-P_{s,\left(r_{B}\right)^{m}} f(x)\right|^{2} d x\right)^{1 / 2},
\end{aligned}
$$

and

$$
\gamma_{3}(f) \equiv \lim _{c \rightarrow \infty} \sup _{\text {ball } B \subset B(0, c)^{\complement}}\left(\frac{1}{|B|[\rho(|B|)]^{2}} \int_{B}\left|f(x)-P_{s,\left(r_{B}\right)^{m}} f(x)\right|^{2} d x\right)^{1 / 2} .
$$

For any function $f \in \mathrm{VMO}_{\rho, L}^{s}\left(\mathbb{R}^{n}\right)$, we define $\|f\|_{\mathrm{VMO}_{\rho, L}^{s}\left(\mathbb{R}^{n}\right)} \equiv\|f\|_{\mathrm{BMO}_{\rho, L}\left(\mathbb{R}^{n}\right)}$.

We next present some properties of the space $\operatorname{VMO}_{\rho, L}^{s}\left(\mathbb{R}^{n}\right)$. To this end, we first recall some notions of tent spaces; see [3, 14, 17].

Let $\Gamma(x) \equiv\left\{(y, t) \in \mathbb{R}_{+}^{n+1}:|x-y|<t\right\}$ denoting the standard cone (of aperture 1) with vertex $x \in \mathbb{R}^{n}$. For any closed set $F$ of $\mathbb{R}^{n}$, denote by $\mathcal{R} F$ the union of all cones with vertices in $F$, i. e., $\mathcal{R} F \equiv \cup_{x \in F} \Gamma(x)$; and for any open set $O$ in $\mathbb{R}^{n}$, denote the tent over $O$ by $\widehat{O}$, which is defined by $\widehat{O} \equiv\left[\mathcal{R}\left(O^{\complement}\right)\right]^{\complement}$.

For all measurable functions $g$ on $\mathbb{R}_{+}^{n+1}$ and all $x \in \mathbb{R}^{n}$, define

$$
\mathcal{A}(g)(x) \equiv\left(\int_{\Gamma(x)}|g(y, t)|^{2} \frac{d y d t}{t^{n+1}}\right)^{1 / 2}
$$


and

$$
\mathcal{C}_{\rho}(g)(x) \equiv \sup _{\text {ball } B \ni x} \frac{1}{\rho(|B|)}\left(\frac{1}{|B|} \int_{\widehat{B}}|g(y, t)|^{2} \frac{d y d t}{t}\right)^{1 / 2} .
$$

For $p \in(0, \infty)$, the tent space $T_{2}^{p}\left(\mathbb{R}_{+}^{n+1}\right)$ is defined to be the set of all measurable functions $g$ on $\mathbb{R}_{+}^{n+1}$ such that $\|g\|_{T_{2}^{p}\left(\mathbb{R}_{+}^{n+1}\right)} \equiv\|\mathcal{A}(g)\|_{L^{p}\left(\mathbb{R}^{n}\right)}<\infty$. The tent space $T_{\omega}\left(\mathbb{R}_{+}^{n+1}\right)$ associated to the function $\omega$ is defined to be the set of all measurable functions $g$ on $\mathbb{R}_{+}^{n+1}$ such that $\mathcal{A}(g) \in L(\omega)$, and its norm is given by

$$
\|g\|_{T_{\omega}\left(\mathbb{R}_{+}^{n+1}\right)} \equiv\|\mathcal{A}(g)\|_{L(\omega)}=\inf \left\{\lambda>0: \int_{\mathbb{R}^{n}} \omega\left(\frac{\mathcal{A}(g)(x)}{\lambda}\right) d x \leq 1\right\}
$$

the space $T_{\omega}^{\infty}\left(\mathbb{R}_{+}^{n+1}\right)$ is defined to be the set of all measurable functions $g$ on $\mathbb{R}_{+}^{n+1}$ such that $\|g\|_{T_{\omega}^{\infty}\left(\mathbb{R}_{+}^{n+1}\right)} \equiv\left\|\mathcal{C}_{\rho}(g)\right\|_{L^{\infty}\left(\mathbb{R}^{n}\right)}<\infty$.

In what follows, let $T_{2, c}^{2}\left(\mathbb{R}_{+}^{n+1}\right)$ denote the set of all $f \in T_{2}^{2}\left(\mathbb{R}_{+}^{n+1}\right)$ with compact supports. Obviously, $T_{2, c}^{2}\left(\mathbb{R}_{+}^{n+1}\right) \subset T_{\omega}^{\infty}\left(\mathbb{R}_{+}^{n+1}\right)$. Similarly, let $T_{\omega, c}^{\infty}\left(\mathbb{R}_{+}^{n+1}\right)$ denote the set of all $f \in T_{\omega}^{\infty}\left(\mathbb{R}_{+}^{n+1}\right)$ with compact supports. It is easy to see that $T_{\omega, c}^{\infty}\left(\mathbb{R}_{+}^{n+1}\right)$ coincides with $T_{2, c}^{2}\left(\mathbb{R}_{+}^{n+1}\right)$. Define $T_{\omega, \mathrm{v}}^{\infty}\left(\mathbb{R}_{+}^{n+1}\right)$ to be the closure of $T_{\omega, c}^{\infty}\left(\mathbb{R}_{+}^{n+1}\right)$ in $T_{\omega}^{\infty}\left(\mathbb{R}_{+}^{n+1}\right)$. On the other hand, let $T_{\omega, 0}^{\infty}\left(\mathbb{R}_{+}^{n+1}\right)$ be the set of all $f \in T_{\omega}^{\infty}\left(\mathbb{R}_{+}^{n+1}\right)$ satisfying $\eta_{1}(f)=\eta_{2}(f)=\eta_{3}(f)=0$, where

$$
\begin{aligned}
& \eta_{1}(f) \equiv \lim _{c \rightarrow 0} \sup _{\text {ball } B: r_{B} \leq c} \frac{1}{\rho(|B|)}\left(\frac{1}{|B|} \int_{\widehat{B}}|f(y, t)|^{2} \frac{d y d t}{t}\right)^{1 / 2}, \\
& \eta_{2}(f) \equiv \lim _{c \rightarrow \infty} \sup _{\text {ball } B: r_{B} \geq c} \frac{1}{\rho(|B|)}\left(\frac{1}{|B|} \int_{\widehat{B}}|f(y, t)|^{2} \frac{d y d t}{t}\right)^{1 / 2},
\end{aligned}
$$

and

$$
\eta_{3}(f) \equiv \lim _{c \rightarrow \infty} \sup _{\text {ball } B \subset B(0, c)^{\mathrm{C}}} \frac{1}{\rho(|B|)}\left(\frac{1}{|B|} \int_{\widehat{B}}|f(y, t)|^{2} \frac{d y d t}{t}\right)^{1 / 2} .
$$

It is easy to see that $T_{\omega, 0}^{\infty}\left(\mathbb{R}_{+}^{n+1}\right)$ is a closed linear subspace of $T_{\omega}^{\infty}\left(\mathbb{R}_{+}^{n+1}\right)$. Moreover, similarly to the proof of Lemma 3.2 in [6], we have the following proposition. We omit the details.

Proposition 3.1 Let $T_{\omega, \mathrm{v}}^{\infty}\left(\mathbb{R}_{+}^{n+1}\right)$ and $T_{\omega, 0}^{\infty}\left(\mathbb{R}_{+}^{n+1}\right)$ be defined as above. Then $T_{\omega, \mathrm{v}}^{\infty}\left(\mathbb{R}_{+}^{n+1}\right)=$ $T_{\omega, 0}^{\infty}\left(\mathbb{R}_{+}^{n+1}\right)$.

Recall that a measure $d \mu$ on $\mathbb{R}_{+}^{n+1}$ is said to be a $\rho$-Carleson measure if

$$
\sup _{B \subset \mathbb{R}^{n}}\left\{\frac{1}{|B|[\rho(|B|)]^{2}} \int_{\widehat{B}}|d \mu|\right\}<\infty
$$

where the supremum is taken over all balls $B$ of $\mathbb{R}^{n}$; see $[14,17]$. 
Let $m$ be the constant in $(2.1), s \geq s_{1} \geq\left\lfloor\frac{n}{m}\left(\frac{1}{\widetilde{p}_{0}(\omega)}-1\right)\right\rfloor$, where $\widetilde{p}_{0}(\omega)$ is the critical lower type index of $\omega$. Let $C_{m, s, s_{1}}$ be a positive constant such that

$$
C_{m, s, s_{1}} \int_{0}^{\infty} t^{m(s+2)} e^{-2 t^{m}}\left(1-e^{-t^{m}}\right)^{s_{1}+1} \frac{d t}{t}=1
$$

The following Lemma 3.1 and Theorem 3.1 were established in [17].

Lemma 3.1 Let L, $\omega$ and $\rho$ be as in Definition 3.1, and $s \geq s_{1} \geq\left\lfloor\frac{n}{m}\left(\frac{1}{\widetilde{p}_{0}(\omega)}-1\right)\right\rfloor$. Suppose that $f \in \mathcal{M}\left(\mathbb{R}^{n}\right)$ such that $\left|Q_{s, t^{m}}\left(I-P_{s_{1}, t^{m}}\right) f(x)\right|^{2} d x d t / t$ is a $\rho$-Carleson measure on $\mathbb{R}_{+}^{n+1}$ and $g \in H_{\omega, L^{*}}\left(\mathbb{R}^{n}\right) \cap L^{2}\left(\mathbb{R}^{n}\right)$. Then

$$
\int_{\mathbb{R}^{n}} f(x) g(x) d x=C_{m, s, s_{1}} \int_{\mathbb{R}_{+}^{n+1}} Q_{s, t^{m}}\left(I-P_{s_{1}, t^{m}}\right) f(x) Q_{t^{m}}^{*} g(x) \frac{d x d t}{t} .
$$

Theorem 3.1 Let L, $\omega$ and $\rho$ be as in Definition 3.1, and $s \geq s_{1} \geq\left\lfloor\frac{n}{m}\left(\frac{1}{\widetilde{p}_{0}(\omega)}-1\right)\right\rfloor$. Then the following conditions are equivalent:

(a) $f \in \mathrm{BMO}_{\rho, L}\left(\mathbb{R}^{n}\right)$;

(b) $f \in \mathcal{M}\left(\mathbb{R}^{n}\right)$ and $\left|Q_{s, t^{m}}\left(I-P_{s_{1}, t^{m}}\right) f(x)\right|^{2} d x d t / t$ is a $\rho$-Carleson measure on $\mathbb{R}_{+}^{n+1}$. Moreover, $\left\|Q_{s, t^{m}}\left(I-P_{s_{1}, t^{m}}\right) f\right\|_{T_{\omega}^{\infty}\left(\mathbb{R}_{+}^{n+1}\right)}$ is equivalent to $\|f\|_{\mathrm{BMO}_{\rho, L}\left(\mathbb{R}^{n}\right)}$.

We now establish a characterization of the space $\mathrm{VMO}_{\rho, L}^{s}\left(\mathbb{R}^{n}\right)$ via the space $T_{\omega, \mathrm{v}}^{\infty}\left(\mathbb{R}_{+}^{n+1}\right)$ by borrowing some ideas from [6]. We remark that if $\omega(t)=t$ for all $t \in(0, \infty)$, then Theorem 3.2 coincides with Proposition 3.3 in [6].

Theorem 3.2 Let L, $\omega$ and $\rho$ be as in Definition 3.1, $s_{1} \geq s_{0} \geq\left\lfloor\frac{n}{m}\left(\frac{1}{\tilde{p}_{0}(\omega)}-1\right)\right\rfloor$ and $s \geq 2 s_{1}$. Then the following conditions are equivalent:

(a) $f \in \mathrm{VMO}_{\rho, L}^{s_{0}}\left(\mathbb{R}^{n}\right)$;

(b) $f \in \mathcal{M}\left(\mathbb{R}^{n}\right)$ and $Q_{s, t^{m}}\left(I-P_{2 s_{1}, t^{m}}\right) f \in T_{\omega, \mathrm{v}}^{\infty}\left(\mathbb{R}_{+}^{n+1}\right)$.

Proof. We first notice that by the assumption (c), there exists $\epsilon \in\left(n \beta_{1}(\rho), \theta(L)\right)$. Recall that $\beta_{1}(\rho)=1 / p_{0}(\omega)-1$ and $p_{0}(\omega)$ is as in the convention. To see that (a) implies (b), by the fact $\mathrm{VMO}_{\rho, L}^{s_{0}}\left(\mathbb{R}^{n}\right) \subset \mathrm{BMO}_{\rho, L}\left(\mathbb{R}^{n}\right)$ together with Theorem 3.1, we only need to verify $Q_{s, t^{m}}\left(I-P_{2 s_{1}, t^{m}}\right) f \in T_{\omega, \mathrm{v}}^{\infty}\left(\mathbb{R}_{+}^{n+1}\right)$. To this end, we need to show that for all balls $B \equiv B\left(x_{B}, r_{B}\right)$,

$$
\frac{1}{\rho(|B|)|B|^{1 / 2}}\left(\int_{\widehat{B}}\left|Q_{s, t^{m}}\left(I-P_{2 s_{1}, t^{m}}\right) f(x)\right|^{2} \frac{d x d t}{t}\right)^{1 / 2} \lesssim \sum_{k=1}^{\infty} 2^{-k \epsilon} \delta_{k}(f, B),
$$

where $\epsilon \in\left(n \beta_{1}(\rho), \theta(L)\right)$ and

$$
\delta_{k}(f, B) \equiv \sup _{B^{\prime} \subset 2^{k+1} B: r_{B^{\prime}} \in\left[r_{B} / 2,2 r_{B}\right]} \frac{1}{\rho\left(\left|B^{\prime}\right|\right)\left|B^{\prime}\right|^{1 / 2}}\left(\int_{B^{\prime}}\left|f(x)-P_{s_{0},\left(r_{B^{\prime}}\right)^{m}} f(x)\right|^{2} d x\right)^{1 / 2} .
$$


Predual Spaces of Banach Completions of Orlicz-Hardy Spaces

In fact, since $f \in \mathrm{VMO}_{\rho, L}^{s_{0}}\left(\mathbb{R}^{n}\right) \subset \mathrm{BMO}_{\rho, L}\left(\mathbb{R}^{n}\right)$, we have $\delta_{k}(f, B) \leq\|f\|_{\mathrm{BMO}_{\rho, L}\left(\mathbb{R}^{n}\right)}$. Moreover, for each $k \in \mathbb{N}$, we have

$$
\lim _{c \rightarrow 0} \sup _{B: r_{B} \leq c} \delta_{k}(f, B)=\lim _{c \rightarrow \infty} \sup _{B: r_{B} \geq c} \delta_{k}(f, B)=\lim _{c \rightarrow \infty} \sup _{B \subset B(0, c)^{\complement}} \delta_{k}(f, B)=0 .
$$

Then by (3.3) and the dominated convergence theorem for series, we have

$$
\begin{aligned}
& \eta_{1}\left(Q_{s, t^{m}}\left(I-P_{2 s_{1}, t^{m}}\right) f\right) \\
& \quad=\lim _{c \rightarrow 0} \sup _{B: r_{B} \leq c} \frac{1}{\rho(|B|)|B|^{1 / 2}}\left(\int_{\widehat{B}}\left|Q_{s, t^{m}}\left(I-P_{2 s_{1}, t^{m}}\right) f(x)\right|^{2} \frac{d x d t}{t}\right)^{1 / 2} \\
& \quad \lesssim \sum_{k=1}^{\infty} 2^{-k \epsilon} \lim _{c \rightarrow 0} \sup _{B: r_{B} \leq c} \delta_{k}(f, B)=0
\end{aligned}
$$

thus $\eta_{1}\left(Q_{s, t^{m}}\left(I-P_{2 s_{1}, t^{m}}\right) f\right)=0$. Similarly, we have $\eta_{2}\left(Q_{s, t^{m}}\left(I-P_{2 s_{1}, t^{m}}\right) f\right)=0=$ $\eta_{3}\left(Q_{s, t^{m}}\left(I-P_{2 s_{1}, t^{m}}\right) f\right)$, which imply $Q_{s, t^{m}}\left(I-P_{2 s_{1}, t^{m}}\right) f \in T_{\omega, \mathrm{V}}^{\infty}\left(\mathbb{R}_{+}^{n+1}\right)$, and hence (a) implies (b).

Let us now prove (3.3). Notice that

$$
\begin{aligned}
& \frac{1}{\rho(|B|)|B|^{1 / 2}}\left(\int_{\widehat{B}}\left|Q_{s, t^{m}}\left(I-P_{2 s_{1}, t^{m}}\right) f(x)\right|^{2} \frac{d x d t}{t}\right)^{1 / 2} \\
& \leq \frac{1}{\rho(|B|)|B|^{1 / 2}}\left(\int_{\widehat{B}} \mid Q_{s, t^{m}}\left(I-P_{2 s_{1}, t^{m}}\right)\left(I-P_{\left.\left.s_{0},\left(r_{2 B}\right)^{m}\right)\left.f(x)\right|^{2} \frac{d x d t}{t}\right)^{1 / 2}}\right.\right. \\
& \quad+\frac{1}{\rho(|B|)|B|^{1 / 2}}\left(\int_{\widehat{B}}\left|Q_{s, t^{m}}\left(I-P_{2 s_{1}, t^{m}}\right) P_{s_{0},\left(r_{2 B}\right)^{m}} f(x)\right|^{2} \frac{d x d t}{t}\right)^{1 / 2} \\
& \equiv \mathrm{I}+\mathrm{J}
\end{aligned}
$$

Set $U_{1}(B) \equiv 2 B$ and $U_{k}(B) \equiv 2^{k} B \backslash 2^{k-1} B$ when $k \geq 2$. For $k \in \mathbb{N}$, let $b_{k} \equiv[(I-$

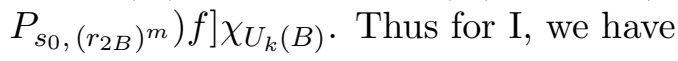

$$
\mathrm{I} \leq \sum_{k=1}^{\infty} \frac{1}{\rho(|B|)|B|^{1 / 2}}\left(\int_{\widehat{B}}\left|Q_{s, t^{m}}\left(I-P_{2 s_{1}, t^{m}}\right) b_{k}(x)\right|^{2} \frac{d x d t}{t}\right)^{1 / 2} \equiv \sum_{k=1}^{\infty} \mathrm{I}_{k} .
$$

When $k=1$, by $(2.4)$, we have

$$
\mathrm{I}_{1} \lesssim \frac{1}{\rho(|B|)|B|^{1 / 2}}\left\|b_{1}\right\|_{L^{2}\left(\mathbb{R}^{n}\right)} \lesssim \delta_{2}(f, B) .
$$

When $k \geq 2$, notice that for any $x \in B$ and $y \in\left(2^{k} B\right)^{\complement},|x-y| \gtrsim 2^{k} r_{B}$. Thus, by (2.3), we obtain

$$
\begin{aligned}
\left|Q_{s, t^{m}}\left(I-P_{2 s_{1}, t^{m}}\right) b_{k}(x)\right| & \lesssim \int_{U_{k}(B)} \frac{t^{\epsilon}}{(t+|x-y|)^{n+\epsilon}}\left|\left(I-P_{s_{0},\left(r_{2 B}\right)^{m}}\right) f(y)\right| d y \\
& \lesssim t^{\epsilon}\left(2^{k} r_{B}\right)^{-n-\epsilon} \int_{2^{k} B}\left|\left(I-P_{s_{0},\left(r_{2 B}\right)^{m}}\right) f(y)\right| d y .
\end{aligned}
$$


To estimate the last term, by the argument in [7, pp.645-646], we have that for any ball $B\left(x_{B}, 2^{k} r_{B}\right)$ with $k \geq 2$, there exists a collection of balls $B_{k, 1}, B_{k, 2}, \cdots, B_{k, N_{k}}$ such that each ball $B_{k, i}$ is of radius $r_{2 B}, B\left(x_{B}, 2^{k} r_{B}\right) \subset \cup_{i=1}^{N_{k}} B_{k, i}$ and $N_{k} \lesssim 2^{n k}$. From this facts and the Hölder inequality, it follows that

$$
\begin{aligned}
\left|Q_{s, t^{m}}\left(I-P_{2 s_{1}, t^{m}}\right) b_{k}(x)\right| & \lesssim \sum_{i=1}^{N_{k}} t^{\epsilon}\left(2^{k} r_{B}\right)^{-n-\epsilon} \int_{B_{k, i}}\left|\left(I-P_{s_{0},\left(r_{2 B}\right)^{m}}\right) f(y)\right| d y \\
& \lesssim\left(\frac{t}{r_{B}}\right)^{\epsilon} 2^{-k \epsilon} \rho(|B|) \delta_{k}(f, B)
\end{aligned}
$$

which yields

$$
\mathrm{I}_{k} \lesssim \frac{1}{|B|^{1 / 2}}\left(\int_{\widehat{B}} t^{2 \epsilon-1} d x d t\right)^{1 / 2} 2^{-k \epsilon}\left(r_{B}\right)^{-\epsilon} \delta_{k}(f, B) \lesssim 2^{-k \epsilon} \delta_{k}(f, B) .
$$

Combining (3.5) and (3.6), we obtain $\mathrm{I} \lesssim \sum_{k=1}^{\infty} 2^{-k \epsilon} \delta_{k}(f, B)$.

To estimate the term J, write $t_{2 B} \equiv\left(r_{2 B}\right)^{m}$ and

$$
\begin{aligned}
I- & P_{2 s_{1}, t^{m}} \\
= & \left(I-e^{-t_{2 B} L}+e^{-t_{2 B} L}-e^{-t^{m} L}\right)^{2 s_{1}+1} \\
= & \left(\sum_{k=0}^{s_{1}} C_{2 s_{1}+1}^{k}(-1)^{k} e^{-k t^{m} L}\left(I-P_{2 s_{1}-s_{0}-k-1, t_{2 B}}\right)\left(I-P_{k-1, t_{2 B}-t^{m}}\right)\right)\left(I-P_{s_{0}, t_{2 B}}\right) \\
& +\left(\sum_{k=s_{1}+1}^{2 s_{1}} C_{2 s_{1}+1}^{k}(-1)^{k} e^{-k t^{m} L}\left(I-P_{2 s_{1}-k, t_{2 B}}\right)\left(I-P_{k-s_{0}-2, t_{2 B}-t^{m}}\right)\right)\left(I-P_{s_{0}, t_{2 B}-t^{m}}\right) \\
& \equiv \Psi_{1}(L)\left(I-P_{s_{0},\left(r_{2 B}\right)^{m}}\right)+\Psi_{2}(L)\left(I-P_{s_{0},\left(r_{2 B}\right)^{m}-t^{m}}\right),
\end{aligned}
$$

where $t \in\left(0, r_{B}\right)$ and $C_{2 s_{1}+1}^{k}$ denotes the combinatorial number. Further, by $(2.3)$, we see that $k_{t, r_{B}}$, the kernel of $Q_{s, t^{m}} P_{s_{0},\left(r_{2 B}\right)^{m}} \Psi_{1}(L)$, satisfies that for all $x, y \in \mathbb{R}^{n}$,

$$
\left|k_{t, r_{B}}(x, y)\right| \lesssim\left(\frac{t}{r_{B}}\right)^{m(s+1)} \frac{\left(r_{B}\right)^{\epsilon}}{\left(r_{B}+|x-y|\right)^{n+\epsilon}} .
$$

By this and some computation similar to the estimate for $\mathrm{I}_{k}$, we obtain that for all $x \in B$,

$$
\begin{aligned}
& \left|Q_{s, t^{m}} P_{s_{0},\left(r_{2 B}\right)^{m}} \Psi_{1}(L)\left(I-P_{s_{0},\left(r_{2 B}\right)^{m}}\right) f(x)\right| \\
& \lesssim \sum_{k=1}^{\infty}\left|Q_{s, t^{m}} P_{s_{0},\left(r_{2 B}\right)^{m}} \Psi_{1}(L)\left[\chi_{U_{k}(B)}\left(I-P_{s_{0},\left(r_{2 B}\right)^{m}}\right) f\right](x)\right| \\
& \lesssim\left(\frac{t}{r_{B}}\right)^{m(s+1)} \sum_{k=1}^{\infty} 2^{-k \epsilon} \rho(|B|) \delta_{k}(f, B) .
\end{aligned}
$$

Similarly, we have that for all $x \in B$,

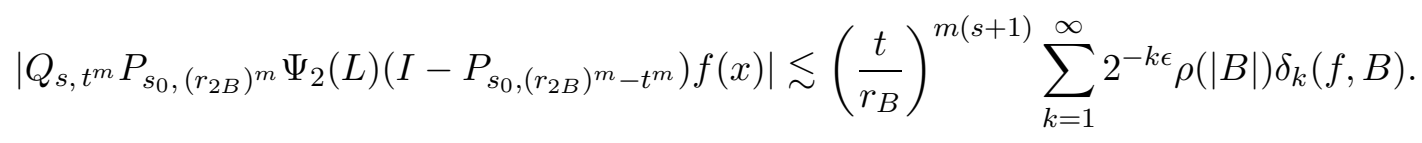


The above two estimates yield

$$
\mathrm{J} \lesssim \frac{1}{|B|^{1 / 2}}\left[\int_{\widehat{B}}\left(\frac{t}{r_{B}}\right)^{2 m(s+1)} \frac{d x d t}{t}\right]^{1 / 2} \sum_{k=1}^{\infty} 2^{-k \epsilon} \delta_{k}(f, B) \lesssim \sum_{k=1}^{\infty} 2^{-k \epsilon} \delta_{k}(f, B) .
$$

The estimates for I and J give (3.3). Thus (a) implies (b).

Conversely, if (b) holds, by Theorem 3.1 , we see that $f \in \mathrm{BMO}_{\rho, L}\left(\mathbb{R}^{n}\right)$. Notice that

$$
\begin{aligned}
& \frac{1}{\rho(|B|)|B|^{1 / 2}}\left(\int_{B}\left|f(x)-P_{s_{0},\left(r_{B}\right)^{m}} f(x)\right|^{2} d x\right)^{1 / 2} \\
& \quad=\sup _{\|g\|_{L^{2}(B)} \leq 1} \frac{1}{\rho(|B|)|B|^{1 / 2}}\left|\int_{B} f(x)\left(I-P_{s_{0},\left(r_{B}\right)^{m}}^{*}\right) g(x) d x\right| .
\end{aligned}
$$

Then by Lemma 3.1, we obtain

$$
\begin{aligned}
& \left|\int_{B} f(x)\left(I-P_{s_{0},\left(r_{B}\right)^{m}}^{*}\right) g(x) d x\right| \\
& \quad=\left|C_{m, s, 2 s_{1}} \int_{\mathbb{R}_{+}^{n+1}} Q_{s, t^{m}}\left(I-P_{2 s_{1}, t^{m}}\right) f(x) Q_{t^{m}}^{*}\left(I-P_{\left.s_{0}, r_{B}\right)^{m}}^{*}\right) g(x) \frac{d x d t}{t}\right| \\
& \quad \lesssim \int_{\widehat{4 B}}\left|Q_{s, t^{m}}\left(I-P_{2 s_{1}, t^{m}}\right) f(x) Q_{t^{m}}^{*}\left(I-P_{s_{0},\left(r_{B}\right)^{m}}^{*}\right) g(x)\right| \frac{d x d t}{t}+\sum_{k=2}^{\infty} \int_{2^{k+1} B} \widehat{2^{k^{k} B}} \cdots \\
& \quad \equiv \mathrm{A}_{1}+\sum_{k=2}^{\infty} \mathrm{A}_{k} .
\end{aligned}
$$

Then the Hölder inequality and (2.4) yield

$$
\begin{aligned}
\mathrm{A}_{1} & \leq\left(\int_{\widehat{4 B}}\left|Q_{s, t^{m}}\left(I-P_{2 s_{1}, t^{m}}\right) f(x)\right|^{2} \frac{d x d t}{t}\right)^{1 / 2}\left(\int_{\widehat{4 B}}\left|Q_{t^{m}}^{*}\left(I-P_{s_{0},\left(r_{B}\right)^{m}}^{*}\right) g(x)\right|^{2} \frac{d x d t}{t}\right)^{1 / 2} \\
& \lesssim\left(\int_{\widehat{4 B}}\left|Q_{s, t^{m}}\left(I-P_{2 s_{1}, t^{m}}\right) f(x)\right|^{2} \frac{d x d t}{t}\right)^{1 / 2} \cdot
\end{aligned}
$$

When $k \geq 2$, notice that

$$
\begin{aligned}
& Q_{t^{m}}^{*}\left(I-P_{s_{0},\left(r_{B}\right)^{m}}^{*}\right) g=t^{m} L^{*} e^{-t^{m} L^{*}}\left(I-e^{-\left(r_{B}\right)^{m} L^{*}}\right)^{s_{0}+1} g \\
& =\int_{0}^{\left(r_{B}\right)^{m}} \cdots \int_{0}^{\left(r_{B}\right)^{m}} \frac{t^{m}}{\left(t^{m}+r_{1}+\cdots+r_{s_{0}+1}\right)^{s_{0}+2}} Q_{s_{0}+1, t^{m}+r_{1}+\cdots+r_{s_{0}+1}}^{*} g d r_{1} \cdots d r_{s_{0}+1} .
\end{aligned}
$$

From (2.3), it is easy to deduce that $q_{s_{0}+1, t^{m}+r_{1}+\cdots+r_{s_{0}+1}}$, the kernel of $Q_{s_{0}+1, t^{m}+r_{1}+\cdots+r_{s_{0}+1}}^{*}$, satisfies that for all $x, y \in \mathbb{R}^{n}$,

$$
\left|q_{s_{0}+1, t^{m}+r_{1}+\cdots+r_{s_{0}+1}}(x, y)\right| \lesssim \frac{\left(t^{m}+r_{1}+\cdots+r_{s_{0}+1}\right)^{\epsilon / m}}{(t+|x-y|)^{n+\epsilon}}
$$


where $\epsilon \in\left(n \beta_{1}(\rho), \theta(L)\right)$. Since $(x, t) \in \widehat{2^{k+1} B} \backslash \widehat{2^{k} B}$ and $y \in B$, we have $|x-y|+t \sim 2^{k} r_{B}$. Moreover, notice that $\epsilon>n \beta_{1}(\rho)$, thus $\epsilon^{\prime} \equiv\left(\epsilon-n \beta_{1}(\rho)\right) / 2>0$. By the Hölder inequality, we have that for all $(x, t) \in \widehat{2^{k+1} B} \backslash \widehat{2^{k} B}$,

$$
\begin{aligned}
\mid Q_{t^{m}}^{*} & \left(I-P_{\left.s_{0},\left(r_{B}\right)^{m}\right) g(x) \mid}^{\left(r_{B}\right)^{m}} \cdots \int_{0}^{\left(r_{B}\right)^{m}} \int_{B} \frac{t^{m}\left(t^{m}+r_{1}+\cdots+r_{s_{0}+1}\right)^{\epsilon / m}}{\left(t^{m}+r_{1}+\cdots+r_{s_{0}+1}\right)^{s_{0}+2}(t+|x-y|)^{n+\epsilon}}\right. \\
& \lesssim \int_{0}^{\left(r^{\prime}\right.} \cdots d g(y) \mid d y d r_{1} \cdots d r_{s_{1}+1}^{\left(r_{B}\right)^{m}} t^{m-m\left(1-\epsilon^{\prime} / m\right)}\left(r_{1} \cdots r_{s_{0}+1}\right)^{-1+\frac{\epsilon-\epsilon^{\prime}}{m\left(s_{0}+1\right)}} d r_{1} \cdots d r_{s_{1}+1} \\
& \lesssim \frac{\|g\|_{L^{1}(B)}}{\left(2^{k} r_{B}\right)^{n+\epsilon}} \int_{0}^{\left(r_{B}\right.} \cdots \int_{0} \\
& \lesssim\left(2^{k} r_{B}\right)^{-n-\epsilon}|B|^{1 / 2} t^{\epsilon^{\prime}}\left(r_{B}\right)^{\epsilon-\epsilon^{\prime}},
\end{aligned}
$$

which implies that

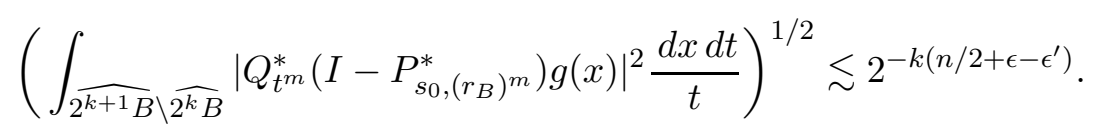

From this together with the Hölder inequality, it follows that

$$
\begin{aligned}
\mathrm{A}_{k} & \lesssim 2^{-k\left(n / 2+\epsilon-\epsilon^{\prime}\right)}\left(\int_{\widehat{2^{k+1} B} \widehat{2^{k} B}}\left|Q_{s, t^{m}}\left(I-P_{2 s_{1}, t^{m}}\right) f(x)\right|^{2} \frac{d x d t}{t}\right)^{1 / 2} \\
& \lesssim 2^{-k\left(\epsilon-n \beta_{1}(\rho)\right) / 2} \frac{|B|^{1 / 2} \rho(|B|)}{\left|2^{k} B\right|^{1 / 2} \rho\left(\left|2^{k} B\right|\right)}\left(\int_{2^{\widehat{k+1} B} \backslash 2^{k} B}\left|Q_{s, t^{m}}\left(I-P_{2 s_{1}, t^{m}}\right) f(x)\right|^{2} \frac{d x d t}{t}\right)^{1 / 2} .
\end{aligned}
$$

Combine the estimates of $\mathrm{A}_{k}$, we finally obtain that

$$
\frac{1}{\rho(|B|)|B|^{1 / 2}}\left(\int_{B}\left|f(x)-P_{s_{0},\left(r_{B}\right)^{m}} f(x)\right|^{2} d x\right)^{1 / 2} \lesssim \sum_{k=1}^{\infty} 2^{-k\left(\epsilon-n \beta_{1}(\rho)\right)} \sigma_{k}(f, B),
$$

where

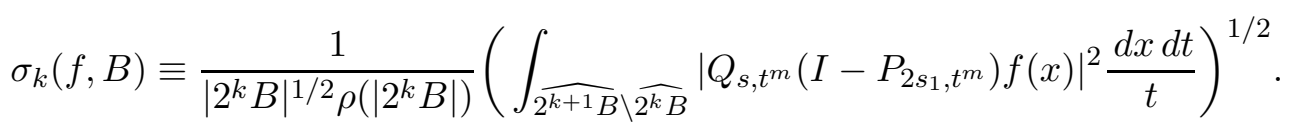

Since $Q_{s, t^{m}}\left(I-P_{2 s_{1}, t^{m}}\right) f \in T_{\omega, \mathrm{v}}^{\infty}\left(\mathbb{R}_{+}^{n+1}\right)$, by Proposition 3.1, for each $k \in \mathbb{N}$, we have

$$
\lim _{c \rightarrow 0} \sup _{B: r_{B} \leq c} \sigma_{k}(f, B)=\lim _{c \rightarrow \infty} \sup _{B: r_{B} \geq c} \sigma_{k}(f, B)=\lim _{c \rightarrow \infty} \sup _{B \subset B(0, c)^{\complement}} \sigma_{k}(f, B)=0 .
$$

Then similarly to the proof of (3.4), we obtain that $\gamma_{1}(f)=\gamma_{2}(f)=\gamma_{3}(f)=0$, which imply that $f \in \mathrm{VMO}_{\rho, L}^{s_{0}}\left(\mathbb{R}^{n}\right)$. This finishes the proof of Theorem 3.2.

Remark 3.1 From Theorem 3.2, it is easy to deduce that if $s \geq s_{0} \geq\left\lfloor\frac{n}{m}\left(\frac{1}{\widetilde{p}_{0}(\omega)}-1\right)\right\rfloor$, then $\mathrm{VMO}_{\rho, L}^{s_{0}}\left(\mathbb{R}^{n}\right)=\mathrm{VMO}_{\rho, L}^{s}\left(\mathbb{R}^{n}\right)$. Hence, in what follows, we denote the space $\operatorname{VMO}_{\rho, L}^{s_{0}}\left(\mathbb{R}^{n}\right)$ simply by $\operatorname{VMO}_{\rho, L}\left(\mathbb{R}^{n}\right)$. 
Now, let $L=\Delta$ be the Laplacian operator on $\mathbb{R}^{n}, p \in(0,1]$ and $\omega(t)=t^{p}$ for all $t \in(0, \infty)$. It was proved in [17] that the space $\mathrm{BMO}_{\rho, L}\left(\mathbb{R}^{n}\right)$ coincides with the space $\Lambda_{n(1 / p-1)}\left(\mathbb{R}^{n}\right)$. We now show that the space $\operatorname{VMO}_{\rho, L}\left(\mathbb{R}^{n}\right)$ and the space $\lambda_{n(1 / p-1)}\left(\mathbb{R}^{n}\right)$ coincide.

Recall that the space $\lambda_{n(1 / p-1)}\left(\mathbb{R}^{n}\right)$ is defined to be the closure of $\mathcal{S}\left(\mathbb{R}^{n}\right)$ (the space of Schwartz functions) in the norm of the Lipschitz space $\Lambda_{n(1 / p-1)}\left(\mathbb{R}^{n}\right)$; see [16, 20, 23]. Let $\psi \in \mathcal{S}\left(\mathbb{R}^{n}\right)$ and $\int_{\mathbb{R}^{n}} \psi(x) x^{\nu} d x=0$ for all $\nu \in \mathbb{Z}_{+}^{n},|\nu| \leq\lfloor n(1 / p-1)\rfloor$ and $\psi_{t}(x)=t^{-n} \psi(x / t)$ for all $x \in \mathbb{R}^{n}$ and $t \in(0, \infty)$. Wang [23] established the following characterization of the space $\lambda_{n(1 / p-1)}\left(\mathbb{R}^{n}\right)$.

Theorem 3.3 Let $p \in(0,1]$ and $\psi$ be as above. A function $f \in \lambda_{n(1 / p-1)}\left(\mathbb{R}^{n}\right)$ if and only if $f * \psi_{t} \in T_{\omega}^{\infty}\left(\mathbb{R}_{+}^{n+1}\right)$ and $\eta_{1}\left(f * \psi_{t}\right)=0$, where $\eta_{1}\left(f * \psi_{t}\right)$ is defined as in (3.1).

Corollary 3.1 Let $L=\Delta, p \in(0,1]$ and $\omega(t)=t^{p}$ for all $t \in(0, \infty)$. Then the space $\mathrm{VMO}_{\rho, L}\left(\mathbb{R}^{n}\right)$ coincides with the space $\lambda_{n(1 / p-1)}\left(\mathbb{R}^{n}\right)$ with equivalent norms.

Proof. Since $L=\Delta$, we then have $m=2$ and $\theta(L)=\infty$. Let $s_{1} \geq\left\lfloor\frac{n}{2}\left(\frac{1}{p}-1\right)\right\rfloor$, $s \geq 2\left(s_{1}+1\right)$ and $f \in \mathrm{VMO}_{\rho, L}\left(\mathbb{R}^{n}\right)$. By Theorem 3.2, we have $Q_{s, t^{m}}\left(I-P_{2 s_{1}, t^{m}}\right) f \in$ $T_{\omega, \mathrm{v}}^{\infty}\left(\mathbb{R}_{+}^{n+1}\right) \subset T_{\omega}^{\infty}\left(\mathbb{R}_{+}^{n+1}\right)$, which together with Proposition 3.1 further implies that

$$
\eta_{1}\left(Q_{s, t^{m}}\left(I-P_{2 s_{1}, t^{m}}\right) f\right)=0 .
$$

Moreover, if we let $\ell \in \mathbb{Z}_{+}$and $\mathcal{P}_{\ell}$ denote the set of all polynomials with degree no more than $\ell$, then $\left(I-P_{2 s_{1}, t^{m}}\right)(g)=0$ for all $g \in \mathcal{P}_{2 s_{1}}\left(\mathbb{R}^{n}\right)$. Thus, by Theorem 3.1, we have $\mathrm{VMO}_{\rho, L}\left(\mathbb{R}^{n}\right) \subset \lambda_{n(1 / p-1)}\left(\mathbb{R}^{n}\right)$.

Conversely, we first point out that it is easy to show that the space $C_{c}^{\infty}\left(\mathbb{R}^{n}\right)$ (the space of all $C^{\infty}\left(\mathbb{R}^{n}\right)$ functions with compact support) is dense in $\lambda_{n(1 / p-1)}\left(\mathbb{R}^{n}\right)$. To prove $\lambda_{n(1 / p-1)}\left(\mathbb{R}^{n}\right) \subset \mathrm{VMO}_{\rho, L}\left(\mathbb{R}^{n}\right)$, by the completeness of these spaces, it suffices to verify that $C_{c}^{\infty}\left(\mathbb{R}^{n}\right) \subset \mathrm{VMO}_{\rho, L}\left(\mathbb{R}^{n}\right)$. Suppose that $f \in C_{c}^{\infty}\left(\mathbb{R}^{n}\right)$. Then $f \in \Lambda_{n(1 / p-1)}\left(\mathbb{R}^{n}\right)=$ $\operatorname{BMO}_{\rho, L}\left(\mathbb{R}^{n}\right)$. Thus, we only need to show that $\gamma_{1}(f)=\gamma_{2}(f)=\gamma_{3}(f)=0$.

Since $\theta(L)=\infty$, we can take $s \geq\left\lfloor n\left(\frac{1}{p}-1\right)\right\rfloor$ and $\epsilon \in(s+1, \infty)$. Then for any $P_{s} \in \mathcal{P}_{s}\left(\mathbb{R}^{n}\right)$ and any ball $B \equiv B\left(x_{B}, r_{B}\right) \subset \mathbb{R}^{n}$, we have

$$
\int_{B}\left|f(x)-P_{s,\left(r_{B}\right)^{m}} f(x)\right|^{2} d x=\int_{B}\left|\left(I-P_{s,\left(r_{B}\right)^{m}}\right)\left(f-P_{s}\right)(x)\right|^{2} d x .
$$

For any $x \in \mathbb{R}^{n}$, write

$$
f(x)=\sum_{|\nu| \leq s} \frac{D^{\nu}(f)\left(x_{B}\right)}{\nu !}\left(x-x_{B}\right)^{\nu}+\sum_{|\nu|=s+1} \frac{D^{\nu}(f)\left(y_{\nu}\right)}{\nu !}\left(x-x_{B}\right)^{\nu},
$$

where for $\nu=\left(\nu_{1}, \cdots, \nu_{n}\right) \in\left(\mathbb{Z}_{+}\right)^{n}, D^{\nu}=\left(\frac{\partial}{\partial x_{1}}\right)^{\nu_{1}} \cdots\left(\frac{\partial}{\partial x_{n}}\right)^{\nu_{n}}$ and $y_{\nu}=\mu x+(1-\mu) x_{B}$ for certain $\mu \in(0,1)$. Let

$$
P_{s}(x)=\sum_{|\nu| \leq s} \frac{D^{\nu}(f)\left(x_{B}\right)}{\nu !}\left(x-x_{B}\right)^{\nu}
$$


By (2.8), $\epsilon>s+1$ and the fact $f \in C_{c}^{\infty}\left(\mathbb{R}^{n}\right)$, we have

$$
\int_{B}\left|f(x)-P_{s}(x)\right|^{2} d x \lesssim \sum_{|\nu|=s+1} \int_{B}\left|\left(x-x_{B}\right)^{\nu}\right|^{2} d x \lesssim\left(r_{B}\right)^{2 s+n+2},
$$

and for any $x \in B$,

$$
\begin{aligned}
& \left|P_{s,\left(r_{B}\right)^{m}}\left(f-P_{s}\right)(x)\right| \\
& \quad \lesssim\left(r_{B}\right)^{-n} \int_{2 B}\left|\left(f-P_{s}\right)(y)\right| d y+\sum_{k=1}^{\infty} \int_{2^{k+1} B \backslash 2^{k} B} \frac{\left(r_{B}\right)^{\epsilon}}{\left(2^{k} r_{B}\right)^{n+\epsilon}}\left|\left(f-P_{s}\right)(y)\right| d y \\
& \quad \lesssim\left(r_{B}\right)^{s+1}+\sum_{k=1}^{\infty} \frac{\left(r_{B}\right)^{\epsilon}}{\left(2^{k} r_{B}\right)^{n+\epsilon}} \sum_{|\nu|=s+1} \int_{2^{k+1} B \backslash 2^{k} B}\left|\left(y-x_{B}\right)^{\nu}\right| d y \\
& \quad \lesssim\left(r_{B}\right)^{s+1}+\sum_{k=1}^{\infty} \frac{\left(r_{B}\right)^{\epsilon}}{\left(2^{k} r_{B}\right)^{n+\epsilon}}\left(2^{k+1} r_{B}\right)^{n+s+1} \lesssim\left(r_{B}\right)^{s+1},
\end{aligned}
$$

which, together the fact that $s \geq\left\lfloor n\left(\frac{1}{p}-1\right)\right\rfloor$, gives

$$
\lim _{c \rightarrow 0} \sup _{B: r_{B} \leq c} \frac{1}{|B|[\rho(|B|)]^{2}} \int_{B}\left|f(x)-P_{s,\left(r_{B}\right)^{m}} f(x)\right|^{2} d x \lesssim \lim _{r_{B} \rightarrow 0} \frac{1}{|B|^{2 / p-1}}\left(r_{B}\right)^{2 s+2+n}=0 .
$$

Thus $\gamma_{1}(f)=0$.

To see $\gamma_{2}(f)=0$, using the fact $f \in C_{c}^{\infty}\left(\mathbb{R}^{n}\right)$ and (2.8), we have for any $t>0$,

$$
\left\|P_{s, t^{m}} f\right\|_{L^{2}\left(\mathbb{R}^{n}\right)} \lesssim\|f\|_{L^{2}\left(\mathbb{R}^{n}\right)}
$$

Thus

$$
\begin{aligned}
\gamma_{2}(f) & =\lim _{c \rightarrow \infty} \sup _{B: r_{B} \geq c} \frac{1}{|B|[\rho(|B|)]^{2}} \int_{B} \mid f(x)-P_{s,\left.\left(r_{B}\right)^{m} f(x)\right|^{2} d x} \\
& \lesssim \lim _{c \rightarrow \infty} \sup _{B: r_{B} \geq c} \frac{1}{|B|[\rho(|B|)]^{2}}\|f\|_{L^{2}\left(\mathbb{R}^{n}\right)}^{2}=0 .
\end{aligned}
$$

Let us show that $\gamma_{3}(f)=0$. By $\gamma_{2}(f)=0$, we know that for any $\beta>0$, there exists $R_{0}>0$ such that if $r_{B} \geq R_{0}$, then

$$
\frac{1}{|B|[\rho(|B|)]^{2}} \int_{B}\left|f(x)-P_{s,\left(r_{B}\right)^{m}} f(x)\right|^{2} d x<\beta .
$$

Thus we only need to consider the case $r_{B} \in\left(0, R_{0}\right)$. Since $f \in C_{c}^{\infty}\left(\mathbb{R}^{n}\right)$, then there exists $r_{0}>0$ such that $\operatorname{supp} f \subset B\left(0, r_{0}\right)$. Let $k_{0} \in \mathbb{N}$ such that $2^{-k_{0}(\epsilon-s-1)}<\beta$. Taking $c>2^{k_{0}}\left(r_{0}+R_{0}\right)$, we deduce that for any ball $B \subset(B(0, c))^{\complement}$ with $r_{B}<R_{0}$, $\left(2^{k_{0}} B\right) \cap B\left(0, r_{0}\right)=\emptyset$. From this and $(2.8)$, we deduce that for any ball $B \subset(B(0, c))^{\complement}$ with $r_{B} \in\left[1, R_{0}\right)$ and $x \in B$,

$$
\left|P_{s,\left(r_{B}\right)^{m}} f(x)\right| \lesssim \int_{B\left(0, r_{0}\right)} \frac{\left(r_{B}\right)^{\epsilon}}{\left(2^{k_{0}} r_{B}\right)^{n+\epsilon}}|f(y)| d y \lesssim\left(r_{B}\right)^{-n} 2^{-k_{0}(n+\epsilon)}\|f\|_{L^{1}\left(\mathbb{R}^{n}\right)}
$$


which implies that

$$
\frac{1}{|B|[\rho(|B|)]^{2}} \int_{B}\left|f(x)-P_{s,\left(r_{B}\right)^{m}} f(x)\right|^{2} d x \lesssim \int_{B}\left|P_{s,\left(r_{B}\right)^{m}} f(x)\right|^{2} d x \lesssim 2^{-2 k_{0}(n+\epsilon)} \lesssim \beta
$$

When $B \equiv B\left(x_{B}, r_{B}\right) \subset(B(0, c))^{\complement}$ with $r_{B} \in(0,1)$, by the fact $\epsilon>s+1$ and $\left(2^{k_{0}} B\right) \cap$ $B\left(0, r_{0}\right)=\emptyset$, similarly to the proof of $\gamma_{1}(f)=0$ with $P_{s}$ as in (3.7), we have that for any $x \in B$,

$$
\begin{aligned}
& \left|f(x)-P_{s,\left(r_{B}\right)^{m}} f(x)\right|=\left|\left(I-P_{s,\left(r_{B}\right)^{m}}\right)\left(f-P_{s}\right)(x)\right| \\
& \lesssim \sum_{k=k_{0}+1}^{\infty} \sum_{|\nu|=s+1} \int_{2^{k} B \backslash 2^{k-1} B} \frac{\left(r_{B}\right)^{\epsilon}}{\left(2^{k} r_{B}\right)^{n+\epsilon}}\left|\left(y-x_{B}\right)^{\nu}\right| d y \\
& \lesssim \sum_{k=k_{0}+1}^{\infty} 2^{-k(n+\epsilon)}\left(r_{B}\right)^{-n}\left(2^{k} r_{B}\right)^{n+s+1} \lesssim 2^{-k_{0}(\epsilon-s-1)}\left(r_{B}\right)^{s+1},
\end{aligned}
$$

which, together with the fact $s \geq\lfloor n(1 / p-1)\rfloor$, gives

$$
\frac{1}{|B|[\rho(|B|)]^{2}} \int_{B}\left|f(x)-P_{s,\left(r_{B}\right)^{m}} f(x)\right|^{2} d x \lesssim 2^{-k_{0}(\epsilon-s-1)} \frac{\left(r_{B}\right)^{2 s+n+2}}{|B|^{2 / p-1}} \lesssim 2^{-2 k_{0}(\epsilon-s-1)} \lesssim \beta .
$$

Hence for any ball $B \subset(B(0, c))^{\complement}$, we have that

$$
\frac{1}{|B|[\rho(|B|)]^{2}} \int_{B}\left|f(x)-P_{s,\left(r_{B}\right)^{m}} f(x)\right|^{2} d x \lesssim \beta
$$

which, together with the fact that $\beta \rightarrow 0$ induces $c \rightarrow \infty$, implies that $\gamma_{3}(f)=0$. Thus $f \in \mathrm{VMO}_{\rho, L}\left(\mathbb{R}^{n}\right)$. Therefore, $\mathrm{VMO}_{\rho, L}\left(\mathbb{R}^{n}\right)=\lambda_{n(1 / p-1)}\left(\mathbb{R}^{n}\right)$, which completes the proof of Corollary 3.1.

Remark 3.2 If $L=\sqrt{\Delta}$, similarly to the proof of Theorem 3.1, we then have that for $p \in(n /(n+1), 1]$ and $w(t)=t^{p}$ for all $t \in(0, \infty)$, the space $\operatorname{VMO}_{\rho, L}\left(\mathbb{R}^{n}\right)$ coincides with the space $\lambda_{n(1 / p-1)}\left(\mathbb{R}^{n}\right)$ with equivalent norms. We omit the details.

\section{Predual spaces of the spaces $B_{\omega, L}\left(\mathbb{R}^{n}\right)$}

In this section, we study the predual space of the space $B_{\omega, L}\left(\mathbb{R}^{n}\right)$, where $B_{\omega, L}\left(\mathbb{R}^{n}\right)$ is the Banach completion of the space $H_{\omega, L}\left(\mathbb{R}^{n}\right)$; see Definition 4.3 and Theorem 4.4 below. We begin with some notions and known facts on tent spaces.

Recall that a function $a$ on $\mathbb{R}_{+}^{n+1}$ is said to be a $T_{\omega}\left(\mathbb{R}_{+}^{n+1}\right)$-atom if

(i) there exists a ball $B \subset \mathbb{R}^{n}$ such that $\operatorname{supp} a \subset \widehat{B}$;

(ii) $\int_{\widehat{B}}|a(x, t)|^{2} \frac{d x d t}{t} \leq|B|^{-1}[\rho(|B|)]^{-2}$.

The following theorem was established in [14]. 
Theorem 4.1 Let $\omega$ satisfy the assumption (c). Then for any $f \in T_{\omega}\left(\mathbb{R}_{+}^{n+1}\right)$, there exist a sequence of $T_{\omega}\left(\mathbb{R}_{+}^{n+1}\right)$-atoms $\left\{a_{j}\right\}_{j}$ and a sequence of numbers $\left\{\lambda_{j}\right\}_{j} \subset \mathbb{C}$ such that

$$
f(x, t)=\sum_{j} \lambda_{j} a_{j}(x, t)
$$

for all $(x, t) \in \mathbb{R}_{+}^{n+1}$. Moreover, there exists a positive constant $C$ such that for all $f \in$ $T_{\omega}\left(\mathbb{R}_{+}^{n+1}\right)$,

$$
\Lambda\left(\left\{\lambda_{j} a_{j}\right\}_{j}\right) \equiv \inf \left\{\lambda>0: \sum_{j}\left|B_{j}\right| \omega\left(\frac{\left|\lambda_{j}\right|\left\|a_{j}\right\|_{T_{2}^{2}\left(\mathbb{R}_{+}^{n+1}\right)}}{\lambda\left|B_{j}\right|^{1 / 2}}\right) \leq 1\right\} \leq C\|f\|_{T_{\omega}\left(\mathbb{R}_{+}^{n+1}\right)}
$$

where $\widehat{B_{j}}$ appears as the support of $a_{j}$.

Definition 4.1 Let $\omega$ satisfy the assumption (c). The space $\widetilde{T}_{\omega}\left(\mathbb{R}_{+}^{n+1}\right)$ is defined to be the set of all $f=\sum_{j} \lambda_{j} a_{j}$ in $\left(T_{\omega}^{\infty}\left(\mathbb{R}_{+}^{n+1}\right)\right)^{*}$, where $\left\{a_{j}\right\}_{j}$ are $T_{\omega}\left(\mathbb{R}_{+}^{n+1}\right)$-atoms and $\left\{\lambda_{j}\right\}_{j} \in \ell^{1}$. If $f \in \widetilde{T}_{\omega}\left(\mathbb{R}_{+}^{n+1}\right)$, then define

$$
\|f\|_{\widetilde{T}_{\omega}\left(\mathbb{R}_{+}^{n+1}\right)} \equiv \inf \left\{\sum_{j}\left|\lambda_{j}\right|\right\}
$$

where the infimum is taken over all possible decompositions of $f$ as above.

By [15, Lemma 3.1], $\widetilde{T}_{\omega}\left(\mathbb{R}_{+}^{n+1}\right)$ is a Banach space. Moreover, we have the following lemma, which implies that $T_{\omega}\left(\mathbb{R}_{+}^{n+1}\right)$ is dense in $\widetilde{T}_{\omega}\left(\mathbb{R}_{+}^{n+1}\right)$. Thus, in this sense, $\widetilde{T}_{\omega}\left(\mathbb{R}_{+}^{n+1}\right)$ is called the Banach completion of the space $T_{\omega}\left(\mathbb{R}_{+}^{n+1}\right)$; see also [16, 20, 23].

Lemma 4.1 Let $\omega$ satisfy the assumption (c). Then there exists a positive constant $C$ such that for all $f \in T_{\omega}\left(\mathbb{R}_{+}^{n+1}\right), f \in \widetilde{T}_{\omega}\left(\mathbb{R}_{+}^{n+1}\right)$ and

$$
\|f\|_{\widetilde{T}_{\omega}\left(\mathbb{R}_{+}^{n+1}\right)} \leq C\|f\|_{T_{\omega}\left(\mathbb{R}_{+}^{n+1}\right)}
$$

Proof. Let $f \in T_{\omega}\left(\mathbb{R}_{+}^{n+1}\right)$. By Theorem 4.1, there exists $T_{\omega}\left(\mathbb{R}_{+}^{n+1}\right)$-atoms $\left\{a_{j}\right\}_{j}$ and $\left\{\lambda_{j}\right\}_{j} \subset \mathbb{C}$ such that (4.1) and (4.2) hold. Further, by the proof of Theorem 4.1 in [14], we may choose $a_{j}$ such that $\left\|a_{j}\right\|_{T_{2}^{2}\left(\mathbb{R}_{+}^{n+1}\right)}=\left|B_{j}\right|^{-1 / 2} \rho\left(\left|B_{j}\right|\right)^{-1}$ for each $j$, where $\operatorname{supp} a_{j} \subset \widehat{B_{j}}$; see also [3].

For any $L \in \mathbb{N}$, set $\sigma_{L} \equiv \sum_{|j| \leq L}\left|\lambda_{j}\right|$. Since $\omega$ is of upper type 1 , by this together with $\rho(t)=t^{-1} / \omega^{-1}\left(t^{-1}\right)$, we obtain

$$
\sum_{j}\left|B_{j}\right| \omega\left(\frac{\left|\lambda_{j}\right||| a_{j} \|_{T_{2}^{2}\left(\mathbb{R}_{+}^{n+1}\right)}}{\sigma_{L}\left|B_{j}\right|^{1 / 2}}\right) \gtrsim \sum_{|j| \leq L}\left|B_{j}\right| \omega\left(\frac{1}{\left|B_{j}\right| \rho\left(\left|B_{j}\right|\right)}\right) \frac{\left|\lambda_{j}\right|}{\sigma_{L}} \gtrsim 1,
$$

which implies that

$$
\sum_{|j| \leq L}\left|\lambda_{j}\right| \lesssim \Lambda\left(\left\{\lambda_{j} a_{j}\right\}_{j}\right) \lesssim\|f\|_{T_{\omega}\left(\mathbb{R}_{+}^{n+1}\right)}
$$


Letting $L \rightarrow \infty$, we further obtain

$$
\|f\|_{\widetilde{T}_{\omega}\left(\mathbb{R}_{+}^{n+1}\right)} \leq \sum_{j}\left|\lambda_{j}\right| \lesssim\|f\|_{T_{\omega}\left(\mathbb{R}_{+}^{n+1}\right)}
$$

which completes the proof of Lemma 4.1.

Lemma 4.2 Let $\omega$ satisfy the assumption (c). Then $\left(\widetilde{T}_{\omega}\left(\mathbb{R}_{+}^{n+1}\right)\right)^{*}=T_{\omega}^{\infty}\left(\mathbb{R}_{+}^{n+1}\right)$ via the pairing

$$
\langle f, g\rangle=\int_{\mathbb{R}_{+}^{n+1}} f(x, t) g(x, t) \frac{d x d t}{t}
$$

for all $f \in \widetilde{T}_{\omega}\left(\mathbb{R}_{+}^{n+1}\right)$ and $g \in T_{\omega}^{\infty}\left(\mathbb{R}_{+}^{n+1}\right)$.

Proof. The facts that $\left(T_{\omega}\left(\mathbb{R}_{+}^{n+1}\right)\right)^{*}=T_{\omega}^{\infty}\left(\mathbb{R}_{+}^{n+1}\right)$ (see [17]) and $T_{\omega}\left(\mathbb{R}_{+}^{n+1}\right) \subset \widetilde{T}_{\omega}\left(\mathbb{R}_{+}^{n+1}\right)$ imply that $\left(\widetilde{T}_{\omega}\left(\mathbb{R}_{+}^{n+1}\right)\right)^{*} \subset T_{\omega}^{\infty}\left(\mathbb{R}_{+}^{n+1}\right)$.

Conversely, let $g \in T_{\omega}^{\infty}\left(\mathbb{R}_{+}^{n+1}\right)$. Then for any $f \in \widetilde{T}_{\omega}\left(\mathbb{R}_{+}^{n+1}\right)$, choose a sequence $T_{\omega}$-atoms $\left\{a_{j}\right\}_{j}$ and $\left\{\lambda_{j}\right\}_{j} \in \ell^{1}$ such that $f=\sum_{j} \lambda_{j} a_{j}$ in $\left(T_{\omega}^{\infty}\left(\mathbb{R}_{+}^{n+1}\right)\right)^{*}$ and $\sum_{j}\left|\lambda_{j}\right| \lesssim\|f\|_{\widetilde{T}_{\omega}\left(\mathbb{R}_{+}^{n+1}\right)}$. Thus by the Hölder inequality, we obtain

$$
\begin{aligned}
|\langle f, g\rangle| & \leq \sum_{j}\left|\lambda_{j}\right| \int_{\mathbb{R}_{+}^{n+1}}\left|a_{j}(x, t) g(x, t)\right| \frac{d x d t}{t} \\
& \leq\|g\|_{T_{\omega}^{\infty}\left(\mathbb{R}_{+}^{n+1}\right)} \sum_{j}\left|\lambda_{j}\right| \lesssim\|g\|_{T_{\omega}^{\infty}\left(\mathbb{R}_{+}^{n+1}\right)}\|f\|_{\widetilde{T}_{\omega}\left(\mathbb{R}_{+}^{n+1}\right)},
\end{aligned}
$$

which implies that $g \in\left(\widetilde{T}_{\omega}\left(\mathbb{R}_{+}^{n+1}\right)\right)^{*}$, and thus completes the proof of Lemma 4.2.

Recall that $T_{\omega, c}^{\infty}\left(\mathbb{R}_{+}^{n+1}\right)$ denotes the set of all functions in $T_{\omega}^{\infty}\left(\mathbb{R}_{+}^{n+1}\right)$ with compact supports.

Lemma 4.3 Let $\omega$ satisfy the assumption (c). If $f \in \widetilde{T}_{\omega}\left(\mathbb{R}_{+}^{n+1}\right)$, then

$$
\|f\|_{\widetilde{T}_{\omega}\left(\mathbb{R}_{+}^{n+1}\right)}=\sup _{g \in T_{\omega, c}^{\infty}\left(\mathbb{R}_{+}^{n+1}\right),\|g\|_{T_{\omega}^{\infty}\left(\mathbb{R}_{+}^{n+1}\right)} \leq 1}\left|\int_{\mathbb{R}_{+}^{n+1}} f(x, t) g(x, t) \frac{d x d t}{t}\right| .
$$

Proof. Let $f \in \widetilde{T}_{\omega}\left(\mathbb{R}_{+}^{n+1}\right)$. By Lemma 4.2, we obtain

$$
\|f\|_{\widetilde{T}_{\omega}\left(\mathbb{R}_{+}^{n+1}\right)}=\sup _{\|g\|_{T_{\omega}^{\infty}\left(\mathbb{R}_{+}^{n+1}\right)} \leq 1}\left|\int_{\mathbb{R}_{+}^{n+1}} f(x, t) g(x, t) \frac{d x d t}{t}\right| .
$$

For any $\beta>0$, there exists $g \in T_{\omega}^{\infty}\left(\mathbb{R}_{+}^{n+1}\right)$ with $\|g\|_{T_{\omega}^{\infty}\left(\mathbb{R}_{+}^{n+1}\right)} \leq 1$ such that

$$
\left|\int_{\mathbb{R}_{+}^{n+1}} f(x, t) g(x, t) \frac{d x d t}{t}\right| \geq\|f\|_{\widetilde{T}_{\omega}\left(\mathbb{R}_{+}^{n+1}\right)}-\frac{\beta}{2} .
$$


Notice here $f g \in L^{1}\left(\mathbb{R}_{+}^{n+1}\right)$. Let $O_{k} \equiv\{(x, t):|x|<k, 1 / k<t<k\}$. Then there exists $k \in \mathbb{N}$ such that

$$
\left|\int_{\mathbb{R}_{+}^{n+1}} f(x, t) g(x, t) \chi_{O_{k}}(x, t) \frac{d x d t}{t}\right| \geq\|f\|_{\widetilde{T}_{\omega}\left(\mathbb{R}_{+}^{n+1}\right)}-\beta .
$$

Obviously, $g \chi_{O_{k}} \in T_{\omega, c}^{\infty}\left(\mathbb{R}_{+}^{n+1}\right)$. Thus (4.3) holds, which completes the proof of Lemma 4.3 .

The following lemma is a slight modification of [4, Lemma 4.2]; see also [20, 23]. We omit the details here.

Lemma 4.4 Let $\omega$ satisfy the assumption (c). Suppose that $\left\{f_{k}\right\}_{k=1}^{\infty}$ is a bounded family of functions in $\widetilde{T}_{\omega}\left(\mathbb{R}_{+}^{n+1}\right)$. Then there exist $f \in \widetilde{T}_{\omega}\left(\mathbb{R}_{+}^{n+1}\right)$ and a subsequence $\left\{f_{k_{j}}\right\}_{j}$ such that for all $g \in T_{\omega, c}^{\infty}\left(\mathbb{R}_{+}^{n+1}\right)$,

$$
\lim _{j \rightarrow \infty} \int_{\mathbb{R}_{+}^{n+1}} f_{k_{j}}(x, t) g(x, t) \frac{d x d t}{t}=\int_{\mathbb{R}_{+}^{n+1}} f(x, t) g(x, t) \frac{d x d t}{t} .
$$

Theorem 4.2 Let $\omega$ satisfy the assumption (c). Then $\left(T_{\omega, \mathrm{v}}^{\infty}\left(\mathbb{R}_{+}^{n+1}\right)\right)^{*}$, the dual space of the space $T_{\omega, \mathrm{v}}^{\infty}\left(\mathbb{R}_{+}^{n+1}\right)$, coincides with $\widetilde{T}_{\omega}\left(\mathbb{R}_{+}^{n+1}\right)$ in the following sense:

(i) For any $g \in \widetilde{T}_{\omega}\left(\mathbb{R}_{+}^{n+1}\right)$, the linear functional $\ell$, which is defined by

$$
\ell(f) \equiv \int_{\mathbb{R}_{+}^{n+1}} f(x, t) g(x, t) \frac{d x d t}{t},
$$

is well defined on $T_{\omega, \mathrm{v}}^{\infty}\left(\mathbb{R}_{+}^{n+1}\right)$ and $\|\ell\|_{\left(T_{\omega, \mathrm{v}}^{\infty}\left(\mathbb{R}_{+}^{n+1}\right)\right)^{*}} \leq C\|g\|_{\widetilde{T}_{\omega}\left(\mathbb{R}_{+}^{n+1}\right)}$, where $C$ is a positive constant independent of $g$.

(ii) Conversely, for any $\ell \in\left(T_{\omega, \mathrm{v}}^{\infty}\left(\mathbb{R}_{+}^{n+1}\right)\right)^{*}$, there exists $g \in \widetilde{T}_{\omega}\left(\mathbb{R}_{+}^{n+1}\right)$ such that (4.4) holds for all $f \in T_{\omega, \mathrm{v}}^{\infty}\left(\mathbb{R}_{+}^{n+1}\right)$ and $\|g\|_{\widetilde{T}_{\omega}\left(\mathbb{R}_{+}^{n+1}\right)} \leq C\|\ell\|_{\left(T_{\omega, \mathrm{v}}^{\infty}\left(\mathbb{R}_{+}^{n+1}\right)\right)^{*}}$, where $C$ is independent of $\ell$.

Proof. By Lemma 4.2, we obtain $\left(\widetilde{T}_{\omega}\left(\mathbb{R}_{+}^{n+1}\right)\right)^{*}=T_{\omega}^{\infty}\left(\mathbb{R}_{+}^{n+1}\right) \supset T_{\omega, \mathrm{v}}^{\infty}\left(\mathbb{R}_{+}^{n+1}\right)$, which further implies that $\widetilde{T}_{\omega}\left(\mathbb{R}_{+}^{n+1}\right) \subset\left(\widetilde{T}_{\omega}\left(\mathbb{R}_{+}^{n+1}\right)\right)^{* *} \subset\left(T_{\omega, \mathrm{v}}^{\infty}\left(\mathbb{R}_{+}^{n+1}\right)\right)^{*}$.

Conversely, let $\ell \in\left(T_{\omega, \mathrm{v}}^{\infty}\left(\mathbb{R}_{+}^{n+1}\right)\right)^{*}$. Notice that for any $f \in T_{2, c}^{2}\left(\mathbb{R}_{+}^{n+1}\right)$, we may assume that $\operatorname{supp} f \subset K$, where $K$ is a compact set in $\mathbb{R}_{+}^{n+1}$. Then we have

$$
\|f\|_{T_{\omega, \mathrm{v}}^{\infty}\left(\mathbb{R}_{+}^{n+1}\right)}=\|f\|_{T_{\omega}^{\infty}\left(\mathbb{R}_{+}^{n+1}\right)} \leq C(K)\|f\|_{T_{2}^{2}\left(\mathbb{R}_{+}^{n+1}\right)} .
$$

Thus, $\ell$ induces a bounded linear functional on $T_{2, c}^{2}\left(\mathbb{R}_{+}^{n+1}\right)$. Let $O_{k}$ be as in the proof of Lemma 4.3. By the Riesz theorem, there exists a unique $g_{k} \in L^{2}\left(O_{k}\right)$ such that for all $f \in L^{2}\left(O_{k}\right)$

$$
\ell(f)=\int_{\mathbb{R}_{+}^{n+1}} f(x, t) g_{k}(x, t) \frac{d x d t}{t}
$$


Obviously, $g_{k+1} \chi_{O_{k}}=g_{k}$ for all $k \in \mathbb{N}$. Let $g \equiv g_{1} \chi_{O_{1}}+\sum_{k=2}^{\infty} g_{k} \chi_{O_{k} \backslash O_{k-1}}$. Then $g \in$ $L_{\text {loc }}^{2}\left(\mathbb{R}_{+}^{n+1}\right)$ and for any $f \in T_{2, c}^{2}\left(\mathbb{R}_{+}^{n+1}\right)$, we have

$$
\ell(f)=\int_{\mathbb{R}_{+}^{n+1}} f(y, t) g(y, t) \frac{d y d t}{t} .
$$

Set $\widetilde{g}_{k} \equiv g \chi_{O_{k}}$. Then for each $k \in \mathbb{N}$, obviously, we have $\widetilde{g}_{k} \in T_{2, c}^{2}\left(\mathbb{R}_{+}^{n+1}\right) \subset T_{\omega}\left(\mathbb{R}_{+}^{n+1}\right) \subset$ $\widetilde{T}_{\omega}\left(\mathbb{R}_{+}^{n+1}\right)$. Then by Lemma 4.3 , we have

$$
\begin{aligned}
\left\|\widetilde{g}_{k}\right\|_{\widetilde{T}_{\omega}\left(\mathbb{R}_{+}^{n+1}\right)} & \sup _{f \in T_{\omega, c}^{\infty}\left(\mathbb{R}_{+}^{n+1}\right),\|f\|_{T_{\omega}^{\infty}\left(\mathbb{R}_{+}^{n+1}\right)} \leq 1}\left|\int_{\mathbb{R}_{+}^{n+1}} f(x, t) g(x, t) \chi_{O_{k}}(x, t) \frac{d x d t}{t}\right| \\
& =\sup _{f \in T_{\omega, c}^{\infty}\left(\mathbb{R}_{+}^{n+1}\right),\|f\|_{T_{\omega}^{\infty}\left(\mathbb{R}_{+}^{n+1}\right)} \leq 1}\left|\ell\left(f \chi_{O_{k}}\right)\right| \\
& \leq \sup _{f \in T_{\omega, c}^{\infty}\left(\mathbb{R}_{+}^{n+1}\right),\|f\|_{T_{\omega}^{\infty}\left(\mathbb{R}_{+}^{n+1}\right)} \leq 1}\|\ell\|_{\left(T_{\omega, \mathrm{v}}^{\infty}\left(\mathbb{R}_{+}^{n+1}\right)\right)^{*}}\|f\|_{T_{\omega}^{\infty}\left(\mathbb{R}_{+}^{n+1}\right)} \leq\|\ell\|_{\left(T_{\omega, \mathrm{v}}^{\infty}\left(\mathbb{R}_{+}^{n+1}\right)\right)^{*} .}
\end{aligned}
$$

Then by Lemma 4.4 , there exists $\widetilde{g} \in \widetilde{T}_{\omega}\left(\mathbb{R}_{+}^{n+1}\right)$ and $\left\{\widetilde{g}_{k_{j}}\right\}_{j} \subset\left\{\widetilde{g}_{k}\right\}_{k}$ such that for all $f \in T_{\omega, c}^{\infty}\left(\mathbb{R}_{+}^{n+1}\right)$,

$$
\lim _{j \rightarrow \infty} \int_{\mathbb{R}_{+}^{n+1}} f(x, t) \widetilde{g}_{k_{j}}(x, t) \frac{d x d t}{t}=\int_{\mathbb{R}_{+}^{n+1}} f(x, t) \widetilde{g}(x, t) \frac{d x d t}{t} .
$$

On the other hand, notice that for sufficient large $k_{j}$, we have

$$
\ell(f)=\int_{\mathbb{R}_{+}^{n+1}} f(x, t) g(x, t) \frac{d x d t}{t}=\int_{\mathbb{R}_{+}^{n+1}} f(x, t) \widetilde{g}_{k_{j}}(x, t) \frac{d x d t}{t}=\int_{\mathbb{R}_{+}^{n+1}} f(x, t) \widetilde{g}(x, t) \frac{d x d t}{t},
$$

which implies that $g=\widetilde{g}$ almost everywhere, hence $g \in \widetilde{T}_{\omega}\left(\mathbb{R}_{+}^{n+1}\right)$. By a density argument, we know that (4.4) also holds for $g$ and all $f \in T_{\omega, \mathrm{v}}^{\infty}\left(\mathbb{R}_{+}^{n+1}\right)$.

Now, let $L_{c}^{2}\left(\mathbb{R}_{+}^{n+1}\right)$ denote the set of $L^{2}\left(\mathbb{R}_{+}^{n+1}\right)$ functions with compact supports. Then for all $f \in L_{c}^{2}\left(\mathbb{R}_{+}^{n+1}\right)$, define $\pi_{L, s, s_{1}}$ by setting, for all $x \in \mathbb{R}^{n}$,

$$
\pi_{L, s, s_{1}}(f)(x) \equiv C_{m, s, s_{1}} \int_{0}^{\infty} Q_{s, t^{m}}\left(I-P_{s_{1}, t^{m}}\right)(f(\cdot, t))(x) \frac{d t}{t},
$$

where $C_{m, s, s_{1}}$ is the same as in (3.2). From (2.4), it is easy to deduce that $\pi_{L, s, s_{1}}$ is well defined on $L_{c}^{2}\left(\mathbb{R}_{+}^{n+1}\right)$; indeed, by the Hölder inequality, for any $f \in L_{c}^{2}\left(\mathbb{R}_{+}^{n+1}\right)$, we have $\pi_{L, s, s_{1}}(f) \in L^{2}\left(\mathbb{R}^{n}\right)$.

The following notion of molecules is introduced in [17]; see also [1, 25].

Definition 4.2 Let $\omega$ satisfy the assumption (c) and $s \geq s_{1} \geq\left\lfloor\frac{n}{m}\left(\frac{1}{\widetilde{p}_{0}(\omega)}-1\right)\right\rfloor$. A function $\alpha$ on $\mathbb{R}^{n}$ is said to be an $\left(\omega, s, s_{1}\right)$-molecule if there exists a $T_{\omega}\left(\mathbb{R}_{+}^{n+1}\right)$-atom a such that for all $x \in \mathbb{R}^{n}$,

$$
\alpha(x) \equiv \pi_{L, s, s_{1}}(a)(x)=C_{m, s, s_{1}} \int_{0}^{\infty} Q_{s, t^{m}}\left(I-P_{s_{1}, t^{m}}\right)(a(\cdot, t))(x) \frac{d t}{t} .
$$


The following result is essentially Theorem 4.3 and Theorem 4.6 in [17].

Theorem 4.3 Let $\omega$ satisfy the assumption (c) and $s \geq s_{1} \geq\left\lfloor\frac{n}{m}\left(\frac{1}{\tilde{p}_{0}(\omega)}-1\right)\right\rfloor$. Then for all $f \in H_{\omega, L}\left(\mathbb{R}^{n}\right)$, there exist a family $\left\{\alpha_{k}\right\}_{k}$ of $\left(\omega, s, s_{1}\right)$-molecules and $\left\{\lambda_{k}\right\}_{k} \subset \mathbb{C}$ such that

$$
f=\sum_{k} \lambda_{k} \alpha_{k}
$$

in $H_{\omega, L}\left(\mathbb{R}^{n}\right)$. Furthermore, if we define

$$
\widetilde{\Lambda}\left(\left\{\lambda_{k} \alpha_{k}\right\}_{k}\right) \equiv \inf \left\{\Lambda\left(\left\{\mu_{k} a_{k}\right\}_{k}\right)\right\}
$$

where the infimum is taken over all possible $T_{\omega}\left(\mathbb{R}_{+}^{n+1}\right)$-atoms $\left\{a_{k}\right\}_{k}$ and $\left\{\mu_{k}\right\}_{k} \subset \mathbb{C}$ such that $\sum_{k} \mu_{k} \pi_{L, s, s_{1}}\left(a_{k}\right)=\sum_{k} \lambda_{k} \alpha_{k}$ in $H_{\omega, L}\left(\mathbb{R}^{n}\right)$, then there exists a positive constant $C$ independent of $f$ such that $\widetilde{\Lambda}\left(\left\{\lambda_{k} \alpha_{k}\right\}_{k}\right) \leq C\|f\|_{H_{\omega, L}\left(\mathbb{R}^{n}\right)}$.

Conversely, suppose that $\left\{\alpha_{k}\right\}_{k}$ is a family of $\left(\omega, s, s_{1}\right)$-molecules and $\left\{\lambda_{k}\right\}_{k} \subset \mathbb{C}$ such that $\Lambda\left(\left\{\lambda_{k} a_{k}\right\}_{k}\right)<\infty$ with $\alpha_{k}=\pi_{L, s, s_{1}}\left(a_{k}\right)$ for all $k$. Then $\sum_{k} \lambda_{k} \alpha_{k} \in H_{\omega, L}\left(\mathbb{R}^{n}\right)$ with

$$
\left\|\sum_{k} \lambda_{k} \alpha_{k}\right\|_{H_{\omega, L}\left(\mathbb{R}^{n}\right)} \leq C \widetilde{\Lambda}\left(\left\{\lambda_{k} \alpha_{k}\right\}_{k}\right),
$$

where $C$ is a positive constant independent of $\left\{\lambda_{k}\right\}_{k}$ and $\left\{\alpha_{k}\right\}_{k}$.

Let us now introduce the Banach completion of the space $H_{\omega, L}\left(\mathbb{R}^{n}\right)$.

Definition 4.3 Let the assumptions (a), (b) and (c) hold and $s \geq s_{1} \geq\left\lfloor\frac{n}{m}\left(\frac{1}{\tilde{p}_{0}(\omega)}-1\right)\right\rfloor$. The space $B_{\omega, L}^{s, s_{1}}\left(\mathbb{R}^{n}\right)$ is defined to be the set of all $f=\sum_{j} \lambda_{j} \alpha_{j}$ in $\left(\mathrm{BMO}_{\rho, L^{*}}\left(\mathbb{R}^{n}\right)\right)^{*}$, where $\left\{\lambda_{j}\right\}_{j} \in \ell^{1}$ and $\left\{\alpha_{j}\right\}_{j}$ are $\left(\omega, s, s_{1}\right)$-molecules. If $f \in B_{\omega, L}^{s, s_{1}}\left(\mathbb{R}^{n}\right)$, define

$$
\|f\|_{B_{\omega, L}^{s, s_{1}}\left(\mathbb{R}^{n}\right)}=\inf \left\{\sum_{j}\left|\lambda_{j}\right|\right\}
$$

where the infimum is taken over all the possible decompositions of $f$ as above.

By [15, Lemma 3.1] again, we see that $B_{\omega, L}^{s, s_{1}}\left(\mathbb{R}^{n}\right)$ is a Banach space. Similarly to the proof of Lemma 4.1, we have the following embedding of $H_{\omega, L}\left(\mathbb{R}^{n}\right)$ into $B_{\omega, L}^{s, s_{1}}\left(\mathbb{R}^{n}\right)$. We omit the details here.

Lemma 4.5 Let the assumptions (a), (b) and (c) hold and $s \geq s_{1} \geq\left\lfloor\frac{n}{m}\left(\frac{1}{\tilde{p}_{0}(\omega)}-1\right)\right\rfloor$. Then there exists a positive constant $C$ such that for all $f \in H_{\omega, L}\left(\mathbb{R}^{n}\right), f \in B_{\omega, L}^{s, s_{1}}\left(\mathbb{R}^{n}\right)$ and

$$
\|f\|_{B_{\omega, L}^{s, s_{1}}\left(\mathbb{R}^{n}\right)} \leq C\|f\|_{H_{\omega, L}\left(\mathbb{R}^{n}\right) .}
$$

As a corollary of Lemma 4.5, we have the following basic properties of the space $B_{\omega, L}^{s, s_{1}}\left(\mathbb{R}^{n}\right)$. 
Proposition 4.1 Let the assumptions (a), (b) and (c) hold and $s \geq s_{1} \geq\left\lfloor\frac{n}{m}\left(\frac{1}{\tilde{p}_{0}(\omega)}-1\right)\right\rfloor$. Then

(i) The space $H_{\omega, L}\left(\mathbb{R}^{n}\right)$ is dense in $B_{\omega, L}^{s, s_{1}}\left(\mathbb{R}^{n}\right)$.

(ii) For any $\widetilde{s} \geq \widetilde{s}_{1} \geq\left\lfloor\frac{n}{m}\left(\frac{1}{\widetilde{p}_{0}(\omega)}-1\right)\right\rfloor$, the spaces $B_{\omega, L}^{s, s_{1}}\left(\mathbb{R}^{n}\right)$ and $B_{\omega, L}^{\widetilde{s} \widetilde{s}_{1}}\left(\mathbb{R}^{n}\right)$ coincide with equivalent norms.

Proof. By Lemma 4.5, we have $H_{\omega, L}\left(\mathbb{R}^{n}\right) \subset B_{\omega, L}\left(\mathbb{R}^{n}\right)$. On the other hand, notice that the set of all finite linear combinations of $\left(\omega, s, s_{1}\right)$-molecules is included in $H_{\omega, L}\left(\mathbb{R}^{n}\right)$ and is also dense in $B_{\omega, L}^{s, s_{1}}\left(\mathbb{R}^{n}\right)$. Thus (i) holds.

To prove (ii), for any $f \in B_{\omega, L}^{s, s_{1}}\left(\mathbb{R}^{n}\right)$, by Definition 4.3 , there exist $\left(\omega, s, s_{1}\right)$-molecules $\left\{\alpha_{j}\right\}_{j}$ and $\left\{\lambda_{j}\right\}_{j} \in \ell^{1}$ such that $f=\sum_{j} \lambda_{j} \alpha_{j}$ in $\left(\operatorname{BMO}_{\rho, L}\left(\mathbb{R}^{n}\right)\right)^{*}$ and

$$
\sum_{j}\left|\lambda_{j}\right| \lesssim\|f\|_{B_{\omega, L}^{s, s_{1}}\left(\mathbb{R}^{n}\right)}
$$

By Theorem 4.3, we have $\alpha_{j} \in H_{\omega, L}\left(\mathbb{R}^{n}\right)$ and $\left\|\alpha_{j}\right\|_{H_{\omega, L}\left(\mathbb{R}^{n}\right)} \lesssim 1$, which together with Lemma 4.5 implies that $\alpha_{j} \in B_{\omega, L}^{\widetilde{s}, \widetilde{s}_{1}}\left(\mathbb{R}^{n}\right)$ and

$$
\left\|\alpha_{j}\right\|_{B_{\omega, L}^{\widetilde{s}, \widetilde{s}_{1}}\left(\mathbb{R}^{n}\right)} \lesssim\left\|\alpha_{j}\right\|_{H_{\omega, L}\left(\mathbb{R}^{n}\right)} \lesssim 1
$$

Then by Definition 4.3 again, there exists $\left(\omega, \widetilde{s}, \widetilde{s}_{1}\right)$-molecules $\left\{\alpha_{j, k}\right\}_{k}$ and $\left\{\lambda_{j, k}\right\}_{k} \in \ell^{1}$ such that $\alpha_{j}=\sum_{k} \lambda_{j, k} \alpha_{j, k}$ in $\left(\mathrm{BMO}_{\rho, L}\left(\mathbb{R}^{n}\right)\right)^{*}$ and $\sum_{k}\left|\lambda_{j, k}\right| \lesssim 1$. Thus, finally, we obtain that

$$
f=\sum_{j} \lambda_{j} \alpha_{j}=\sum_{j} \lambda_{j}\left\{\sum_{k} \lambda_{j, k} \alpha_{j, k}\right\}
$$

in $\left(\mathrm{BMO}_{\rho, L}\left(\mathbb{R}^{n}\right)\right)^{*}$ and

$$
\|f\|_{B_{\omega, L}^{\widetilde{s} \tilde{s}_{1}\left(\mathbb{R}^{n}\right)}} \leq \sum_{j}\left|\lambda_{j}\right|\left\{\sum_{k}\left|\lambda_{j, k}\right|\right\} \lesssim \sum_{j}\left|\lambda_{j}\right| \lesssim\|f\|_{B_{\omega, L}^{s, s_{1}}\left(\mathbb{R}^{n}\right)} .
$$

Thus $B_{\omega, L}^{s, s_{1}}\left(\mathbb{R}^{n}\right) \subset B_{\omega, L}^{\widetilde{s}, \widetilde{s}_{1}}\left(\mathbb{R}^{n}\right)$. By symmetry, we also have $B_{\omega, L}^{\widetilde{s} \widetilde{s}_{1}}\left(\mathbb{R}^{n}\right) \subset B_{\omega, L}^{s, s_{1}}\left(\mathbb{R}^{n}\right)$, which completes the proof of Proposition 4.1.

Proposition 4.1 (ii) implies that the space $B_{\omega, L}^{s, s_{1}}\left(\mathbb{R}^{n}\right)$ with $s, s_{1}$ as in Definition 4.3 is independent of the choices of $s$ and $s_{1}$. Based on this, from now on, for any $s \geq s_{1} \geq$ $\left\lfloor\frac{n}{m}\left(\frac{1}{\widetilde{p}_{0}(\omega)}-1\right)\right\rfloor$, we denote the space $B_{\omega, L}^{s, s_{1}}\left(\mathbb{R}^{n}\right)$ simply by $B_{\omega, L}\left(\mathbb{R}^{n}\right)$. Also, by Proposition 4.1 (i), we call the space $B_{\omega, L}\left(\mathbb{R}^{n}\right)$ the Banach completion of the Orlicz-Hardy space $H_{\omega, L}\left(\mathbb{R}^{n}\right)$; see also Definition 4.1 and its following remarks.

The proof of the following lemma is similar to that of Lemma 4.2. We omit the details.

Lemma 4.6 Let the assumptions (a), (b) and (c) hold. Then $\left(B_{\omega, L}\left(\mathbb{R}^{n}\right)\right)^{*}$ coincides with $\mathrm{BMO}_{\rho, L^{*}}\left(\mathbb{R}^{n}\right)$ in the following sense: 
(i) For any $g \in \mathrm{BMO}_{\rho, L^{*}}\left(\mathbb{R}^{n}\right)$, the linear functional $\ell$, which is initially defined on $B_{\omega, L}\left(\mathbb{R}^{n}\right) \cap L^{2}\left(\mathbb{R}^{n}\right)$ by

$$
\ell(f) \equiv \int_{\mathbb{R}^{n}} f(x) g(x) d x
$$

has an unique extension to $B_{\omega, L}\left(\mathbb{R}^{n}\right)$ with $\|\ell\|_{\left(B_{\omega, L}\left(\mathbb{R}^{n}\right)\right)^{*}} \leq C\|g\|_{\mathrm{BMO}_{\rho, L^{*}}\left(\mathbb{R}^{n}\right)}$, where $C$ is a positive constant independent of $g$.

(ii) Conversely, for any $\ell \in\left(B_{\omega, L}\left(\mathbb{R}^{n}\right)\right)^{*}$, there exists $g \in \mathrm{BMO}_{\rho, L^{*}}\left(\mathbb{R}^{n}\right)$ such that (4.6) holds for all $f \in B_{\omega, L}\left(\mathbb{R}^{n}\right) \cap L^{2}\left(\mathbb{R}^{n}\right)$ and $\|g\|_{\mathrm{BMO}_{\rho, L^{*}}\left(\mathbb{R}^{n}\right)} \leq C\|\ell\|_{\left(B_{\omega, L}\left(\mathbb{R}^{n}\right)\right)^{*}}$, where $C$ is independent of $\ell$.

Lemma 4.7 Let $\omega$ satisfy the assumption (c). Then $T_{2, c}^{2}\left(\mathbb{R}_{+}^{n+1}\right)$ is dense in $\widetilde{T}_{\omega}\left(\mathbb{R}_{+}^{n+1}\right)$.

Proof. Since $T_{\omega}\left(\mathbb{R}_{+}^{n+1}\right)$ is dense in $\widetilde{T}_{\omega}\left(\mathbb{R}_{+}^{n+1}\right)$, to prove the lemma, it suffices to prove that $T_{2, c}^{2}\left(\mathbb{R}_{+}^{n+1}\right)$ is dense in $T_{\omega}\left(\mathbb{R}_{+}^{n+1}\right)$ in the norm $\|\cdot\|_{\widetilde{T}_{\omega}\left(\mathbb{R}_{+}^{n+1}\right)}$.

For any $g \in T_{\omega}\left(\mathbb{R}_{+}^{n+1}\right)$, let $g_{k} \equiv g \chi_{O_{k}}$, where $O_{k}$ is the same as in the proof of Lemma 4.3. Then $g_{k} \in T_{2, c}^{2}\left(\mathbb{R}_{+}^{n+1}\right)$. By the dominated convergence theorem and the continuity of $\omega$, we have that

$$
\lim _{k \rightarrow \infty} \int_{\mathbb{R}^{n}} \omega\left(\mathcal{A}\left(g-g_{k}\right)(x)\right) d x=\int_{\mathbb{R}^{n}} \lim _{k \rightarrow \infty} \omega\left(\mathcal{A}\left(g-g_{k}\right)(x)\right) d x=0,
$$

which implies that $\lim _{k \rightarrow \infty}\left\|g-g_{k}\right\|_{T_{\omega}\left(\mathbb{R}_{+}^{n+1}\right)}=0$. Then, by Lemma 4.1, we have

$$
\left\|g-g_{k}\right\|_{\widetilde{T}_{\omega}\left(\mathbb{R}_{+}^{n+1}\right)} \lesssim\left\|g-g_{k}\right\|_{T_{\omega}\left(\mathbb{R}_{+}^{n+1}\right)} \rightarrow 0,
$$

as $k \rightarrow \infty$, which completes the proof of Lemma 4.7 .

Lemma 4.8 Let the assumptions (a), (b) and (c) hold and $s \geq s_{1} \geq\left\lfloor\frac{n}{m}\left(\frac{1}{\tilde{p}_{0}(\omega)}-1\right)\right\rfloor$. Then the operator $\pi_{L, s, s_{1}}$, initially defined on $L_{c}^{2}\left(\mathbb{R}_{+}^{n+1}\right)$, extends to a bounded linear operator:

(i) from $T_{2}^{p}\left(\mathbb{R}_{+}^{n+1}\right)$ to $L^{p}\left(\mathbb{R}^{n}\right)$, if $p \in(1, \infty)$; or

(ii) from $T_{\omega}\left(\mathbb{R}_{+}^{n+1}\right)$ to $H_{\omega, L}\left(\mathbb{R}^{n}\right)$; or

(iii) from $\widetilde{T}_{\omega}\left(\mathbb{R}_{+}^{n+1}\right)$ to $B_{\omega, L}\left(\mathbb{R}^{n}\right)$.

Proof. Proposition (i) was established in [25] and proposition (ii) was established in [17]. Let us now prove (iii).

Let $f \in T_{2, c}^{2}\left(\mathbb{R}_{+}^{n+1}\right)$. Since $T_{2, c}^{2}\left(\mathbb{R}_{+}^{n+1}\right) \subset T_{\omega}\left(\mathbb{R}_{+}^{n+1}\right)$, by (ii), we have $\pi_{L, s, s_{1}}(f) \in$ $H_{\omega, L}\left(\mathbb{R}^{n}\right) \subset B_{\omega, L}^{s, s_{1}}\left(\mathbb{R}^{n}\right)$. Moreover, since $T_{\omega}\left(\mathbb{R}_{+}^{n+1}\right) \subset \widetilde{T}_{\omega}\left(\mathbb{R}_{+}^{n+1}\right)$, by Definition 4.1 , there exist $T_{\omega}\left(\mathbb{R}_{+}^{n+1}\right)$-atoms $\left\{a_{j}\right\}_{j}$ and $\left\{\lambda_{j}\right\}_{j} \subset \mathbb{C}$ such that $f=\sum_{j} \lambda_{j} a_{j}$ in $\left(T_{\omega}^{\infty}\left(\mathbb{R}_{+}^{n+1}\right)\right)^{*}$ and $\sum_{j}\left|\lambda_{j}\right| \lesssim\|f\|_{\widetilde{T}_{\omega}\left(\mathbb{R}_{+}^{n+1}\right)}$. Thus for any $g \in \mathrm{BMO}_{\rho, L^{*}}\left(\mathbb{R}^{n}\right)$, by Theorem 3.1, we obtain

$$
\left\langle\pi_{L, s, s_{1}}(f), g\right\rangle=\int_{\mathbb{R}_{+}^{n+1}} f(x, t) Q_{s, t^{m}}^{*}\left(I-P_{s_{1}, t^{m}}^{*}\right)(g)(x) \frac{d x d t}{t}
$$


Predual Spaces of Banach Completions of Orlicz-Hardy Spaces

$$
\begin{aligned}
& =\sum_{j} \lambda_{j} \int_{\mathbb{R}_{+}^{n+1}} a_{j}(x, t) Q_{s, t^{m}}^{*}\left(I-P_{s_{1}, t^{m}}^{*}\right)(g)(x) \frac{d x d t}{t} \\
& =\sum_{j} \lambda_{j}\left\langle\pi_{L, s, s_{1}}\left(a_{j}\right), g\right\rangle,
\end{aligned}
$$

which implies that $\pi_{L, s, s_{1}}(f)=\sum_{j} \lambda_{j} \pi_{L, s, s_{1}}\left(a_{j}\right)$ in $\left(\mathrm{BMO}_{\rho, L^{*}}\left(\mathbb{R}^{n}\right)\right)^{*}$ and hence,

$$
\left\|\pi_{L, s, s_{1}}(f)\right\|_{B_{\omega, L}^{s, s_{1}}\left(\mathbb{R}^{n}\right)} \leq \sum_{j}\left|\lambda_{j}\right| \lesssim\|f\|_{\widetilde{T}_{\omega}\left(\mathbb{R}_{+}^{n+1}\right)}
$$

Since by Lemma $4.7, T_{2, c}^{2}\left(\mathbb{R}_{+}^{n+1}\right)$ is dense in $\widetilde{T}_{\omega}\left(\mathbb{R}_{+}^{n+1}\right)$, we then obtain (iii) by a density argument, and thus complete the proof of Lemma 4.8 .

We also define the operator $\pi_{L}$ by setting, for all $f \in L_{c}^{2}\left(\mathbb{R}_{+}^{n+1}\right)$ and $x \in \mathbb{R}^{n}$,

$$
\pi_{L}(f)(x) \equiv C_{m} \int_{0}^{\infty} Q_{t^{m}}(f(\cdot, t))(x) \frac{d t}{t}
$$

where $C_{m}=4 m$. Similarly to (4.5), from (2.4), it is easy to deduce that the operator $\pi_{L}$ is well defined.

Lemma 4.9 Let the assumptions (a), (b) and (c) hold. Then the operator $\pi_{L}$, initially defined on $L_{c}^{2}\left(\mathbb{R}_{+}^{n+1}\right)$, extends to a bounded linear operator:

(i) from $T_{2}^{p}\left(\mathbb{R}_{+}^{n+1}\right)$ to $L^{p}\left(\mathbb{R}^{n}\right)$, if $p \in(1, \infty)$; or

(ii) from $T_{\omega, \mathrm{v}}^{\infty}\left(\mathbb{R}_{+}^{n+1}\right)$ to $\mathrm{VMO}_{\rho, L}\left(\mathbb{R}^{n}\right)$.

Proof. Proposition (i) was established in [12, Lemma 4.3]. To prove (ii), let $s_{1} \geq$ $\left\lfloor\frac{n}{m}\left(\frac{1}{\tilde{p}_{0}(\omega)}-1\right)\right\rfloor, s \geq 2 s_{1}, \epsilon \in\left(n \beta_{1}(\rho), \theta(L)\right)$ and $\widetilde{\epsilon}=\left(\epsilon-n \beta_{1}(\rho)\right) / 2$. Suppose that $f \in T_{\omega, \mathrm{v}}^{\infty}\left(\mathbb{R}_{+}^{n+1}\right)$. To see $\pi_{L}(f) \in \mathrm{VMO}_{\rho, L}\left(\mathbb{R}^{n}\right)$, by Theorem 3.2, it suffices to show that $Q_{s, t^{m}}\left(I-P_{2 s_{1}, t^{m}}\right) \pi_{L}(f) \in T_{\omega, \mathrm{v}}^{\infty}\left(\mathbb{R}_{+}^{n+1}\right)$. We first show that for any ball $B$,

$$
\frac{1}{\rho(|B|)|B|^{1 / 2}}\left(\int_{\widehat{B}}\left|Q_{s, t^{m}}\left(I-P_{2 s_{1}, t^{m}}\right) \pi_{L}(f)(x)\right|^{2} \frac{d x d t}{t}\right)^{1 / 2} \lesssim \sum_{k=1}^{\infty} 2^{-k \widetilde{\epsilon}} \sigma_{k}(f, B),
$$

where

$$
\sigma_{k}(f, B) \equiv \frac{1}{\left|2^{k} B\right|^{1 / 2} \rho\left(\left|2^{k} B\right|\right)}\left(\int_{2^{k+1} B}|f(x, t)|^{2} \frac{d x d t}{t}\right)^{1 / 2} .
$$

Once (4.7) is proved, then by an argument similar to that used in the proof of Theorem 3.2 (see the proof of (3.4)), we obtain that $Q_{s, t^{m}}\left(I-P_{2 s_{1}, t^{m}}\right) \pi_{L}(f) \in T_{\omega, \mathrm{v}}^{\infty}\left(\mathbb{R}_{+}^{n+1}\right)$ and hence $\pi_{L}(f) \in \mathrm{VMO}_{\rho, L}\left(\mathbb{R}^{n}\right)$.

To prove $(4.7)$, let $f_{1} \equiv f \chi_{\widehat{4 B}}$ and $f_{2} \equiv f \chi_{(\widehat{4 B})^{\mathrm{C}}}$. Then

$$
\left(\int_{\widehat{B}}\left|Q_{s, t^{m}}\left(I-P_{2 s_{1}, t^{m}}\right) \pi_{L}(f)(x)\right|^{2} \frac{d x d t}{t}\right)^{1 / 2}
$$




$$
\leq \sum_{i=1}^{2}\left(\int_{\widehat{B}}\left|Q_{s, t^{m}}\left(I-P_{2 s_{1}, t^{m}}\right) \pi_{L}\left(f_{i}\right)(x)\right|^{2} \frac{d x d t}{t}\right)^{1 / 2} \equiv \sum_{i=1}^{2} \mathrm{I}_{i} .
$$

For the term $\mathrm{I}_{1}$, by (2.4) and (i) with $p=2$, we obtain

$$
\mathrm{I}_{1} \lesssim\left\|\pi_{L}\left(f_{1}\right)\right\|_{L^{2}\left(\mathbb{R}^{n}\right)} \lesssim\left\|f_{1}\right\|_{T_{2}^{2}\left(\mathbb{R}_{+}^{n+1}\right)} \sim|2 B|^{1 / 2} \rho(|2 B|) \sigma_{2}(f, B)
$$

To estimate $\mathrm{I}_{2}$, write

$$
\begin{aligned}
Q_{s, t^{m}}\left(I-P_{2 s_{1}, t^{m}}\right) \pi_{L}\left(f_{2}\right)(x) & =\int_{0}^{\infty} Q_{s, t^{m}}\left(I-P_{2 s_{1}, t^{m}}\right) Q_{\nu^{m}}\left(f_{2}\right)(x) \frac{d \nu}{\nu} \\
& \equiv \int_{0}^{\infty} \Psi_{t, \nu}(L)\left(f_{2}\right)(x) \frac{d \nu}{\nu} .
\end{aligned}
$$

It follows from $(2.3)$ that $\psi_{t, \nu}$, the kernel of $\Psi_{t, \nu}(L)$, satisfies that for all $x, y \in \mathbb{R}^{n}$,

$$
\left|\psi_{t, \nu}(x, y)\right| \lesssim \frac{t^{m(s+1)} \nu^{m}}{(t+\nu)^{m(s+2)}} \frac{(t+\nu)^{\epsilon}}{(t+\nu+|x-y|)^{n+\epsilon}} \lesssim \frac{t^{\epsilon-\widetilde{\epsilon}} \nu^{\widetilde{\epsilon}}}{(t+\nu+|x-y|)^{n+\epsilon}}
$$

Moreover, for $k \geq 2$ and $(x, \nu) \in \widehat{2^{k+1} B} \backslash \widehat{2^{k} B}$, we have $t+\nu+|x-y| \sim 2^{k} r_{B}$. By this and the Hölder inequality, we deduce that

$$
\begin{aligned}
& \mathrm{I}_{2} \lesssim \sum_{k=2}^{\infty}\left(\int_{\widehat{B}}\left[\int_{\widehat{2^{k+1} B} \widehat{2^{k} B}}\left|\psi_{t, \nu}(x, y)\right||f(y, \nu)| \frac{d y d \nu}{\nu}\right]^{2} \frac{d x d t}{t}\right)^{1 / 2} \\
& \lesssim \sum_{k=2}^{\infty}\left(\int_{\widehat{B}}\left[\int_{2^{k+1} B \backslash 2^{k} B} \frac{t^{\epsilon-\widetilde{\epsilon}} \nu^{\widetilde{\epsilon}}}{(t+\nu+|x-y|)^{n+\epsilon}}|f(y, \nu)| \frac{d y d \nu}{\nu}\right]^{2} \frac{d x d t}{t}\right)^{1 / 2} \\
& \lesssim \sum_{k=2}^{\infty}\left(\int_{\widehat{B}}\left[\int_{2^{k+1} B \backslash 2^{k} B} \nu^{2 \widetilde{\epsilon}} \frac{d y d \nu}{\nu}\right] t^{2 \epsilon-2 \widetilde{\epsilon}} \frac{d x d t}{t}\right)^{1 / 2}\left(2^{k} r_{B}\right)^{-n-\epsilon}\left|2^{k} B\right|^{1 / 2} \rho\left(\left|2^{k} B\right|\right) \sigma_{k}(f, B) \\
& \lesssim \sum_{k=2}^{\infty}\left[\left(2^{k} r_{B}\right)^{n+2 \widetilde{\epsilon}} r_{B}^{n+2 \epsilon-2 \widetilde{\epsilon}}\right]^{1 / 2}\left(2^{k} r_{B}\right)^{-n / 2-\epsilon} 2^{k n \beta_{1}(\rho)} \rho(|B|) \sigma_{k}(f, B) \\
& \lesssim \sum_{k=2}^{\infty} 2^{-k\left(\epsilon-n \beta_{1}(\rho)\right) / 2} \rho(|B|)|B|^{1 / 2} \sigma_{k}(f, B) .
\end{aligned}
$$

Thus, (4.7) is proved, which completes the proof of Lemma 4.9.

Lemma 4.10 Let the assumptions (a), (b) and (c) hold and $\rho$ be as in (2.10). Then $\mathrm{VMO}_{\rho, L}\left(\mathbb{R}^{n}\right) \cap L^{2}\left(\mathbb{R}^{n}\right)$ is dense in $\operatorname{VMO}_{\rho, L}\left(\mathbb{R}^{n}\right)$.

Proof. Let $s_{1} \geq\left\lfloor\frac{n}{m}\left(\frac{1}{\tilde{p}_{0}(\omega)}-1\right)\right\rfloor$ and $s \geq 2 s_{1}$. Notice that for any $f \in L^{2}\left(\mathbb{R}^{n}\right)$, we have

$$
f=\pi_{L, s, 2 s_{1}}(f)=C_{m, s, 2 s_{1}} \int_{0}^{\infty} Q_{s, t^{m}}\left(I-P_{2 s_{1}, t^{m}}\right) Q_{t^{m}} f \frac{d t}{t}
$$


in $L^{2}\left(\mathbb{R}^{n}\right)$, where $C_{m, s, 2 s_{1}}$ is as in (3.2).

Let $g \in \mathrm{VMO}_{\rho, L}\left(\mathbb{R}^{n}\right)$. For any $f \in H_{\omega, L^{*}}\left(\mathbb{R}^{n}\right) \cap L^{2}\left(\mathbb{R}^{n}\right)$, by Lemma 3.1, we have

$$
\int_{\mathbb{R}^{n}} f(x) g(x) d x=C_{m, s, 2 s_{1}} \int_{\mathbb{R}_{+}^{n+1}} Q_{t^{m}}^{*} f(x) Q_{s, t^{m}}\left(I-P_{2 s_{1}, t^{m}}\right) g(x) \frac{d x d t}{t} .
$$

Since $g \in \mathrm{VMO}_{\rho, L}\left(\mathbb{R}^{n}\right)$, by Theorem 3.2 , we know that $Q_{s, t^{m}}\left(I-P_{2 s_{1}, t^{m}}\right) g \in T_{\omega, \mathrm{v}}^{\infty}\left(\mathbb{R}_{+}^{n+1}\right)$. Let $O_{k}$ be as in the proof of Lemma $4.3, h \equiv Q_{s, t^{m}}\left(I-P_{2 s_{1}, t^{m}}\right) g$ and $h_{k} \equiv \chi_{O_{k}} h$ for each $k \in \mathbb{N}$. Then $h_{k} \in T_{\omega, c}^{\infty}\left(\mathbb{R}_{+}^{n+1}\right)=T_{2, c}^{2}\left(\mathbb{R}_{+}^{n+1}\right)$ and $\left\|h_{k}-h\right\|_{T_{\omega}^{\infty}\left(\mathbb{R}_{+}^{n+1}\right)} \rightarrow 0$, as $k \rightarrow \infty$. Thus by Lemma 4.9 , we have that $\pi_{L}\left(h_{k}\right) \in \operatorname{VMO}_{\rho, L}\left(\mathbb{R}^{n}\right) \cap L^{2}\left(\mathbb{R}^{n}\right)$ and

$$
\left\|\pi_{L}\left(h-h_{k}\right)\right\|_{\mathrm{BMO}_{\rho, L}\left(\mathbb{R}^{n}\right)} \lesssim\left\|\left(h-h_{k}\right)\right\|_{T_{\omega}^{\infty}\left(\mathbb{R}_{+}^{n+1}\right)} \rightarrow 0,
$$

as $k \rightarrow \infty$. Then by the dominated convergence theorem, we further have

$$
\begin{aligned}
\int_{\mathbb{R}^{n}} f(x) g(x) d x & =C_{m, s, 2 s_{1}} \int_{\mathbb{R}_{+}^{n+1}} Q_{t^{m}}^{*} f(x) h(x, t) \frac{d x d t}{t} \\
& =C_{m, s, 2 s_{1}} \lim _{k \rightarrow \infty} \int_{\mathbb{R}_{+}^{n+1}} Q_{t^{m}}^{*} f(x) h_{k}(x, t) \frac{d x d t}{t} \\
& =C_{m, s, 2 s_{1}} \lim _{k \rightarrow \infty} \int_{\mathbb{R}^{n}} f(x) \int_{0}^{\infty} Q_{t^{m}}\left(h_{k}\right)(x) \frac{d x d t}{t} \\
& =\frac{C_{m, s, 2 s_{1}}}{C_{m}} \lim _{k \rightarrow \infty}\left\langle f, \pi_{L}\left(h_{k}\right)\right\rangle=\frac{C_{m, s, 2 s_{1}}}{C_{m}}\left\langle f, \pi_{L}(h)\right\rangle .
\end{aligned}
$$

Since $H_{\omega, L^{*}}\left(\mathbb{R}^{n}\right) \cap L^{2}\left(\mathbb{R}^{n}\right)$ is dense in the space $H_{\omega, L^{*}}\left(\mathbb{R}^{n}\right)$, we then obtain that

$$
g=\frac{C_{m, s, 2 s_{1}}}{C_{m}} \pi_{L}(h)=\frac{C_{m, s, 2 s_{1}}}{C_{m}} \pi_{L}\left(Q_{s, t^{m}}\left(I-P_{2 s_{1}, t^{m}}\right) g\right)
$$

in $\operatorname{VMO}_{\rho, L}\left(\mathbb{R}^{n}\right)$. Let $g_{k} \equiv \frac{C_{m, s, 2 s_{1}}}{C_{m}} \pi_{L}\left(h_{k}\right)$, then by Lemma 4.9 again, we have that $g_{k} \in$ $\mathrm{VMO}_{\rho, L}\left(\mathbb{R}^{n}\right) \cap L^{2}\left(\mathbb{R}^{n}\right)$ and by $(4.8),\left\|g-g_{k}\right\|_{\mathrm{BMO}_{\rho, L}\left(\mathbb{R}^{n}\right)} \rightarrow 0$, as $k \rightarrow \infty$, which completes the proof of Lemma 4.10 .

The following is the main result of this paper. Recall that by Definition 3.1,

$$
\mathrm{VMO}_{\rho, L^{*}}\left(\mathbb{R}^{n}\right) \subset \mathrm{BMO}_{\rho, L^{*}}\left(\mathbb{R}^{n}\right) .
$$

The symbol $\langle\cdot, \cdot\rangle$ in the following theorem means the duality between $\mathrm{BMO}_{\rho, L^{*}}\left(\mathbb{R}^{n}\right)$ and the space $B_{\omega, L}\left(\mathbb{R}^{n}\right)$ in the sense of Lemma 4.6.

Theorem 4.4 Let the assumptions (a), (b) and (c) hold. Then $\left(\mathrm{VMO}_{\rho, L^{*}}\left(\mathbb{R}^{n}\right)\right)^{*}=$ $B_{\omega, L}\left(\mathbb{R}^{n}\right)$ in the following sense:

(i) For any $g \in B_{\omega, L}\left(\mathbb{R}^{n}\right)$, the linear functional $\ell$, which is defined by setting, for all $f \in \mathrm{VMO}_{\rho, L^{*}}\left(\mathbb{R}^{n}\right)$,

$$
\ell(f) \equiv\langle f, g\rangle
$$


Then there exists a positive constant $C$ independent of $g$ such that $\|\ell\|_{\left(\operatorname{VMO}_{\rho, L^{*}}\left(\mathbb{R}^{n}\right)\right)^{*}} \leq$ $C\|g\|_{B_{\omega, L}\left(\mathbb{R}^{n}\right)}$.

(ii) Conversely, for any $\ell \in\left(\mathrm{VMO}_{\rho, L^{*}}\left(\mathbb{R}^{n}\right)\right)^{*}$, there exists $g \in B_{\omega, L}\left(\mathbb{R}^{n}\right)$, such that (4.9) holds and there exists a positive constant $C$ independent of $\ell$ such that $\|g\|_{B_{\omega, L}\left(\mathbb{R}^{n}\right)} \leq$ $C\|\ell\|_{\left(\mathrm{VMO}_{\rho, L^{*}}\left(\mathbb{R}^{n}\right)\right)^{*}}$

Proof. Since $\mathrm{VMO}_{\rho, L^{*}}\left(\mathbb{R}^{n}\right) \subset \mathrm{BMO}_{\rho, L^{*}}\left(\mathbb{R}^{n}\right)$, from Lemma 4.6, it is easy to see that $B_{\omega, L}\left(\mathbb{R}^{n}\right) \subset\left(\mathrm{VMO}_{\rho, L^{*}}\left(\mathbb{R}^{n}\right)\right)^{*}$.

Conversely, let $s_{1} \geq\left\lfloor\frac{n}{m}\left(\frac{1}{\tilde{p}_{0}(\omega)}-1\right)\right\rfloor, s \geq 2 s_{1}$ and $\ell \in\left(\operatorname{VMO}_{\rho, L^{*}}\left(\mathbb{R}^{n}\right)\right)^{*}$. For any $f \in \operatorname{VMO}_{\rho, L^{*}}\left(\mathbb{R}^{n}\right) \cap L^{2}\left(\mathbb{R}^{n}\right)$, by Theorem 3.2 , we have $Q_{s, t^{m}}^{*}\left(I-P_{2 s_{1}, t^{m}}^{*}\right) f \in T_{\omega, \mathrm{v}}^{\infty}\left(\mathbb{R}_{+}^{n+1}\right)$. On the other hand, from Lemma 4.8, it follows that $\ell \circ \pi_{L^{*}} \in\left(T_{\omega, \mathrm{v}}^{\infty}\left(\mathbb{R}_{+}^{n+1}\right)\right)^{*}$. From this together with Theorem 4.2 , we obtain that there exists $g \in \widetilde{T}_{\omega}\left(\mathbb{R}_{+}^{n+1}\right)$ such that

$$
\begin{aligned}
\ell(f) & =\frac{C_{m, s, 2 s_{1}}}{C_{m}} \ell \circ \pi_{L^{*}}\left(Q_{s, t^{m}}^{*}\left(I-P_{2 s_{1}, t^{m}}^{*}\right) f\right) \\
& =\frac{C_{m, s, 2 s_{1}}}{C_{m}} \int_{\mathbb{R}_{+}^{n+1}} Q_{s, t^{m}}^{*}\left(I-P_{2 s_{1}, t^{m}}^{*}\right) f(x) g(x, t) \frac{d x d t}{t} .
\end{aligned}
$$

Since $g \in \widetilde{T}_{\omega}\left(\mathbb{R}_{+}^{n+1}\right)$, there exists $T_{\omega}$-atoms $\left\{a_{j}\right\}_{j}$ and $\{\lambda\}_{j} \subset \mathbb{C}$ such that $g=\sum_{j} \lambda_{j} a_{j}$ in $\left(T_{\omega}^{\infty}\left(\mathbb{R}_{+}^{n+1}\right)\right)^{*}$ and $\sum_{j}\left|\lambda_{j}\right| \lesssim\|g\|_{\widetilde{T}_{\omega}\left(\mathbb{R}_{+}^{n+1}\right)}$. For each $k \in \mathbb{N}$, let $g_{k} \equiv \sum_{j=1}^{k} \lambda_{j} a_{j}$. Then $g_{k} \in$ $T_{2}^{2}\left(\mathbb{R}_{+}^{n+1}\right) \cap T_{\omega}\left(\mathbb{R}_{+}^{n+1}\right)$. Moreover, by Lemma 4.8 , we obtain that $\pi_{L, s, 2 s_{1}}(g) \in B_{\omega, L}\left(\mathbb{R}^{n}\right)$, $\pi_{L, s, 2 s_{1}}\left(g_{k}\right) \in B_{\omega, L}\left(\mathbb{R}^{n}\right) \cap L^{2}\left(\mathbb{R}^{n}\right)$ and

$$
\left\|\pi_{L, s, 2 s_{1}}(g)-\pi_{L, s, 2 s_{1}}\left(g_{k}\right)\right\|_{B_{\omega, L}\left(\mathbb{R}^{n}\right)} \lesssim\left\|g-g_{k}\right\|_{\widetilde{T}_{\omega}\left(\mathbb{R}_{+}^{n+1}\right)} \rightarrow 0
$$

as $k \rightarrow \infty$. Thus by the dominated convergence theorem, we further obtain that

$$
\begin{aligned}
\ell(f) & =\frac{C_{m, s, 2 s_{1}}}{C_{m}} \int_{\mathbb{R}_{+}^{n+1}} Q_{s, t^{m}}^{*}\left(I-P_{2 s_{1}, t^{m}}^{*}\right) f(x) g(x, t) \frac{d x d t}{t} \\
& \sim \lim _{k \rightarrow \infty} \int_{\mathbb{R}_{+}^{n+1}} Q_{s, t^{m}}^{*}\left(I-P_{2 s_{1}, t^{m}}^{*}\right) f(x) g_{k}(x, t) \frac{d x d t}{t} \\
& \sim \lim _{k \rightarrow \infty} \int_{\mathbb{R}^{n}} f(x) \pi_{L, s, 2 s_{1}}\left(g_{k}\right)(x) d x \sim \lim _{k \rightarrow \infty}\left\langle f, \pi_{L, s, 2 s_{1}}\left(g_{k}\right)\right\rangle \\
& \sim \sum_{j} \lambda_{j}\left\langle f, \pi_{L, s, 2 s_{1}}\left(a_{j}\right)\right\rangle \sim\left\langle f, \pi_{L, s, 2 s_{1}}(g)\right\rangle .
\end{aligned}
$$

Then a density argument via Lemma 4.10 completes the proof of Theorem 4.4.

Remark 4.1 By Proposition 4.1 (ii), we know that for any $s \geq s_{1} \geq\left\lfloor\frac{n}{m}\left(\frac{1}{\widetilde{p}_{0}(\omega)}-1\right)\right\rfloor$, $B_{\omega, L}\left(\mathbb{R}^{n}\right)=B_{\omega, L}^{s, s_{1}}\left(\mathbb{R}^{n}\right)$. Thus by Lemma 4.6, (4.9) can be further understood as

$$
\ell(f) \equiv\langle f, g\rangle=\sum_{j} \lambda_{j} \int_{\mathbb{R}^{n}} f(x) \alpha_{j}(x) d x,
$$

where $\left\{\alpha_{j}\right\}_{j}$ is a sequence of $\left(\omega, s, s_{1}\right)$-molecules and $\left\{\lambda_{j}\right\}_{j} \in \ell^{1}$ such that $g=\sum_{j} \lambda_{j} \alpha_{j}$ in $\left(\mathrm{BMO}_{\rho, L^{*}}\left(\mathbb{R}^{n}\right)\right)^{*}$ and $\sum_{j}\left|\lambda_{j}\right| \sim\|g\|_{B_{\omega, L}\left(\mathbb{R}^{n}\right)}$; see also [4, Theorem B]. 


\section{References}

[1] P. Auscher, X. T. Duong and A. McIntosh, Boundedness of Banach space valued singular integral operators and Hardy spaces, Unpublished Manuscript (2005).

[2] S. Blunck and P. C. Kunstmann, Weak type $(p, p)$ estimates for Riesz transforms, Math. Z. 247 (2004), 137-148.

[3] R. R. Coifman, Y. Meyer and E. M. Stein, Some new function spaces and their applications to harmonic analysis, J. Funct. Anal. 62 (1985), 304-335.

[4] R. R. Coifman and G. Weiss, Extensions of Hardy spaces and their use in analysis, Bull. Amer. Math. Soc. 83 (1977), 569-645.

[5] T. Coulhon and X. T. Duong, Maximal regularity and kernel bounds: observations on a theorem by Hieber and Prüss, Adv. Differential Equations 5 (2000), 343-368.

[6] D. Deng, X. T. Duong, L. Song, C. Tan and L. Yan, Functions of vanishing mean oscillation associated with operaptors and applications, Michigan Math. J. (to appear).

[7] D. Deng, X. T. Duong and L. Yan, A characterization of the Morrey-Campanato spaces, Math. Z. 250 (2005), 641-655.

[8] N. Dunford and J. T. Schwartz, Linear operators. Part I. General theory, Reprint of the 1958 original. Wiley Classics Library. A Wiley-Interscience Publication. John Wiley \& Sons, Inc., New York, 1988.

[9] X. T. Duong and A. McIntosh, Singular integral operators with non-smooth kernels on irregular domains, Rev. Mat. Iberoamericana 15 (1999), 233-265.

[10] X. T. Duong, J. Xiao and L. Yan, Old and new Morrey spaces with heat kernel bounds, J. Fourier Anal. Appl. 13 (2007), 87-111.

[11] X. T. Duong and L. Yan, New function spaces of BMO type, the John-Nirenberg inequality, interpolation, and applications, Comm. Pure Appl. Math. 58 (2005), 1375-1420.

[12] X. T. Duong and L. Yan, Duality of Hardy and BMO spaces associated with operators with heat kernel bounds, J. Amer. Math. Soc. 18 (2005), 943-973.

[13] X. T. Duong and L. Yan, New Morrey-Campanato spaces associated with operators and applications, Preprint.

[14] E. Harboure, O. Salinas and B. Viviani, A look at $\mathrm{BMO}_{\phi}(\omega)$ through Carleson measures, J. Fourier Anal. Appl. 13 (2007), 267-284.

[15] S. Hofmann and S. Mayboroda, Hardy and BMO spaces associated to divergence form elliptic operators, Math. Ann. (to appear).

[16] S. Janson, Generalizations of Lipschitz spaces and an application to Hardy spaces and bounded mean oscillation, Duke Math. J. 47 (1980), 959-982.

[17] R. Jiang, D. Yang and Y. Zhou, Orlicz-Hardy spaces associated with operators, Sci. China Ser. A (to appear).

[18] A. McIntosh, Operators which have an $H_{\infty}$ functional calculus, Proc. Centre Math. Anal. Austral. Nat. Univ., 14, Austral. Nat. Univ., Canberra, 1986, 210-231.

[19] E. M. Ouhabaz, Analysis of heat equations on domains, London Math. Soc. Mono. 31, Princeton Univ. Press, Princeton, N. J., 2004.

[20] L. Peng, The dual spaces of $\lambda_{\alpha}\left(\mathbb{R}^{n}\right)$, Thesis in Peking Univ., Beijing, 1981. 
[21] D. Sarason, Functions of vanishing mean oscillation, Trans. Amer. Math. Soc. 207 (1975), 391-405.

[22] B. E. Viviani, An atomic decomposition of the predual of $\operatorname{BMO}(\rho)$, Rev. Mat. Iberoamericana 3 (1987), 401-425.

[23] W. Wang, The predual spaces of tent spaces and some characterizations of $\lambda_{\alpha}\left(\mathbb{R}^{n}\right)$ spaces, Beijing Daxue Xuebao 24 (1988), 535-551.

[24] L. Yan, Littlewood-Paley functions associated to second order elliptic operators, Math. Z. 246 (2004), 655-666.

[25] L. Yan, Classes of Hardy spaces associated with operators, duality theorem and applications, Trans. Amer. Math. Soc. 360 (2008), 4383-4408.

[26] K. Yosida, Functional analysis, Sixth Edition, Spring-Verlag, Berlin, 1978.

Renjin Jiang and Dachun Yang (Corresponding author)

School of Mathematical Sciences, Beijing Normal University, Laboratory of Mathematics and Complex Systems, Ministry of Education, Beijing 100875, People's Republic of China

E-mail addresses: rj-jiang@mail.bnu.edu.cn

dcyang@bnu.edu.cn 